\title{
Interaction of ISPs: Distributed Resource Allocation and Revenue Maximization
}

\author{
Sam C.M. Lee Joe W.J. Jiang Dah-Ming Chiu John C.S. Lui ${ }^{+}$
}

\begin{abstract}
The Internet is a hierarchical architecture comprising heterogeneous entities of privately owned infrastructures, where higher level Internet service providers (ISPs) supply connectivity to the local ISPs and charge the local ISPs for the transit services. One of the challenging problems facing service providers today is how to increase the profitability while maintaining good service qualities as the network scales up. In this work, we seek to understand the fundamental issues on the "interplay" (or interaction) between ISPs at different tiers. While the local ISPs (which we term peers) can communicate with each other by purchasing the connectivity from transit ISPs, there stands an opportunity for them to set up private peering relationships. Under this competitive framework, we explore the issues on (a) impact of peering relationship, (b) resource distribution, (c) revenue maximization, and (d) condition for network upgrade. Firstly, a generalized model is presented to characterize the behaviors of peers and the transit ISP, in which their economic interests are reflected. We study how a peer can distributively determine its optimal peering strategy. Furthermore, we show how a transit ISP is able to utilize the available information to infer its optimal pricing strategy, under which a revenue maximization is achieved. Two distributed algorithms are proposed to help ISPs to provide a fair and efficient bandwidth allocation to peers, avoiding a resource monopolization of the market. Last but not least, we investigate the above issues in a many-peers-region, i.e., when we scale up the network. We provide insightful evidence to show that the ISPs can still gain profits as they upgrade the network infrastructures. Extensive simulations are carried out to support our theoretical claims.
\end{abstract}

Keywords: ISP peering, economic pricing, distributed resource allocation, scalability

\section{Introduction}

One of the challenging problems facing today's Internet Service Providers (ISPs) is how to increase the profitability and at the same time, provide good performance to users as we scale up the network. For the Internet, it is a hierarchical architecture comprising heterogeneous entities of privately owned infrastructures. Generally speaking, the networks can be categorized into two types of service providers: (1) local ISPs which consist of geographically close meshed networks. These local ISPs provide Internet access and connectivity services for consumers within their regions. And (2) large-scaled ISPs which traverse across large geographical distances, providing connectivity among the local ISPs. Figure 1 illustrates what today's Internet looks like. In the figure, one can observe that there are local ISPs which provide Internet services in a small region and transit ISPs which inter-connect these local ISPs.

Sam C.M. Lee, Joe W.J. Jiang and John C.S. Lui are from Department of Computer Science \& Engineering, The Chinese University of Hong Kong. Dah-Ming Chiu is from Department of Information Engineering, The Chinese University of Hong Kong. ${ }^{+}$John C.S. Lui is the contact author, email: cslui@cse.cuhk.edu.hk. 


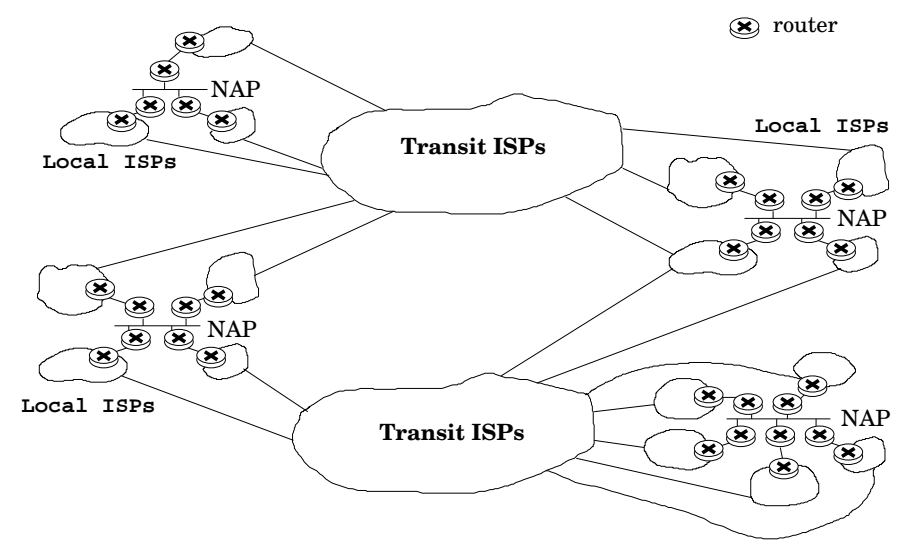

Fig. 1. Today's Internet consisting of multiple transit ISPs and local ISPs.

For the local ISPs, in order to gain the Internet access, a common way is to purchase this service from higher level ISPs (or we called transit ISPs). These transit ISPs set charges for the service provisioning, which depend on the allocated transmission bandwidth as well as the amount of transferred traffic. One important issue is to come up with a good pricing model for the current Internet, especially to reflect the economic roles of different ISPs. Currently, most ISPs adopt a flat rate pricing scheme, i.e., end users pay a fixed amount of money to gain the Internet access in a certain period of time (usually on a monthly basis). Most broadband and ADSL services are examples of this type. Another approach is to charge users by the time they connect to the Internet, following the charging methods employed in the telephony industry. Still, there are ISPs who charge users based on the actual traffic volume transmitted. There are some existing work which investigate the pricing strategy for the service providers. In [6], authors discuss how a provider should price its services differentially based on their characteristics such that prices can match service qualities. Authors in [5] also discuss how to present a cooperative pricing strategy to provide a fair distribution of profits to ISPs.

Besides relying on the transit ISPs for Internet access, local ISPs can also inter-connect their networks together by signing up private peering agreements. For local ISPs which are geographically close to each other, there is an opportunity to exchange information between themselves and bypass the reliance on transit ISPs. One possible way to accomplish this is to establish a private peering link between two parties. In practice, these peering agreements can be quite complicated, involving many business considerations [12][7]. However, the basic nature of the peering relationship is to exchange local traffic between the two local ISPs through the peering link without paying for the traffic transfer. Note that free peering is only one special case of the peering relationship, having charges on peers are also considered in more generalized circumstances. Usually such peering relationship is beneficial to both ISPs since it can provide better performance and at the same time, reduce the operating cost since traffic does not need to go through the transit ISPs. 
There are a number of existing work which explore the economics of network pricing with multiple ISPs on the Internet, recent work being[14], [2], [9], [17]. These authors all investigate a basic question: How to set prices for the Internet services, so as to fairly share revenues among providers, and at the same time encourage the network to grow? Unfortunately, these works underestimate the impact of local peering relationship on the traffic demand, since this will influence the proper pricing strategy to achieve a maximization of ISP's profitability. To bridge this gap, our work aims to seek a fundamental understanding of the interaction between ISPs with peering links. We explore how the peering relationship can affect the service purchasing strategies and pricing strategies played by ISPs. For the ease of presentation, in the rest of this paper, we term the local ISPs simply as peers since they tend to establish peering relationships with each other. Similarly, we refer to the transit ISPs as ISPS. In this work, we are interested to explore the interactions between the connecting peers, as well as the interactions between the peers and the ISP. We also investigate the implications of these interactions.

To communicate with another local ISP, a peer has two options: either to use the connection provided by the transit ISPs, or to use the peering link connecting the two peers. Even given a constant transmission demand, deciding on an appropriate proportion of traffic delivered via these two connections, however, is not a trivial matter. Another factor which makes the decision of traffic allocation difficult is that all peers are rational, i.e., they want to maximize their happiness by transmitting/receiving traffic, and at the same time, they also care about the quality of service they receive and the total payments for consuming the services. Also, one peer's optimal strategy may depend on strategies taken by other peers, as well as the pricing policy employed by the ISP. All these make it a challenging task to come up with an efficient resource allocation policy.

ISP, on the other hand, provides Internet access and connectivity between peers. Its goal is to maximize its own revenue by providing connectivity service. In order to maximize the total profit and attract more potential peers, a good pricing strategy is essential. In general, a transit ISP needs to address the following issues:

- Resource Distribution: how should the ISP sell and allocate its capacity resource to the competing peers, and at the same time, avoid the monopolization of bandwidth resources by a small number of peers?

- Maximization of Revenue: is there a unique price by which the ISP's revenue can be maximized under a homogeneous pricing scheme (i.e., all peers are charged using the same pricing model)? If it exists, how can one find this optimal price?

- Upgrade of Capacity: when more users demand for Internet access, more peers will enter the market. Is there an incentive for the ISP to upgrade the network infrastructures, i.e., increase the backbone capacity to accommodate more peers? Does the increase in revenue compensate for the increased cost 
of deploying new services, or equivalently, does the marginal benefit of the ISP increase as the business is growing?

- Impact of Peering Relationship: a more tricky yet important question is, as the peer population grows, what impact does the private peering relationship have on the ISP's pricing decision?

While these questions have substantial impact and important implications, it is not straightforward to obtain an immediate answer. From the ISP's perspective, it is undesirable that its resource be utilized (or monopolized) by a small number of peers since the ISP wants to achieve customer diversification. To attract or retain a peer for the connectivity service, an ISP has to perform a "fair" resource distribution which avoids resource monopolization. To achieve this goal, the ISP and peers have to exchange traffic information. Note that one has to consider a minimal information exchange due to business confidentiality, as well as the necessity to perform resource allocation in a distributed manner. On the other hand, maximizing its own profit is also an important objective for the ISP. With a particular price offered by the ISP, every connecting peer decides the amount of traffic to send through the transit ISP. The aggregate traffic thus determines the total demand on the ISP link. Setting a lower price attracts more traffic from the peers, but this may lead to traffic congestion. Moreover, a low price does not guarantee the maximization of the ISP's revenue. Setting a higher price, on the other hand, may discourage peers to purchase the ISP service and the traffic demand will decrease, which does not ensure a maximal profit for the ISP as well. Therefore, finding an optimal unit price is an important issue.

The contribution of our paper is to answer the questions listed above. We explore the interplay or interaction between ISPs at different tiers, discussing issues on (a) impact of peering relationship, (b) resource distribution, (c) revenue maximization, and (d) the possibility of network upgrade when we scale up the network.

- We present a generalized model to capture a snapshot of the current Internet, a hierarchy consisting of ISPs of two tiers with peering relationships. We believe this two-tier-interaction represents a basic element of the complicated system, characterizing ISPs' behaviors beyond which their economic interests are reflected.

- We study how a peer can distributively determine its optimal peering strategy by solving a convex optimization problem.

- We propose and compare two distributed algorithms, namely Proportional Share Algorithm (PSA) and Equal Share Algorithm (ESA), to help ISP provide an efficient and fair bandwidth allocation to peers. We show that to avoid monopolization of the market is not a trivial issue.

- We further explore how a transit ISP is able to utilize its available information to infer an optimal pricing strategy under which its revenue maximization can be achieved.

- More importantly, we provide a fundamental understanding of the above problems in a many-users- 
region, i.e., when the network scales up: whether the ISP has any incentives to perform network upgrade, e.g., increase the backbone capacity, so as to adapt more users entering the market? Equivalently, does the marginal profit of an ISP keep increasing as the number of users $n \rightarrow \infty$ ?

The organization of the paper is as follows. In Section II, we present our mathematical model and formulate the optimization problems for a peer and the ISP. In Section III, we show the operating conditions for a peer to obtain the maximum utility under the special case that the traffic demand is constant. In Section IV, we extend the optimization to a general case and derive the operating conditions. In Section $\mathrm{V}$, we propose two algorithms for the ISP to distribute its resource among the peers. We carry out simulations to examine the performance of these two algorithms respectively. In Section VI, we propose a methodology on how an ISP can estimate its optimal pricing strategy to maximize its revenue. We also present an example to illustrate the proposed procedure. In Section VII, we investigate whether the ISP has an incentive to upgrade the network when one scales up the number of peers. We provide simulation results and show that the ISP can benefit from upgrading the network, independent of whether private peering links exist or not. Section VIII presents the related work and Section IX concludes.

\section{The Mathematical Model}
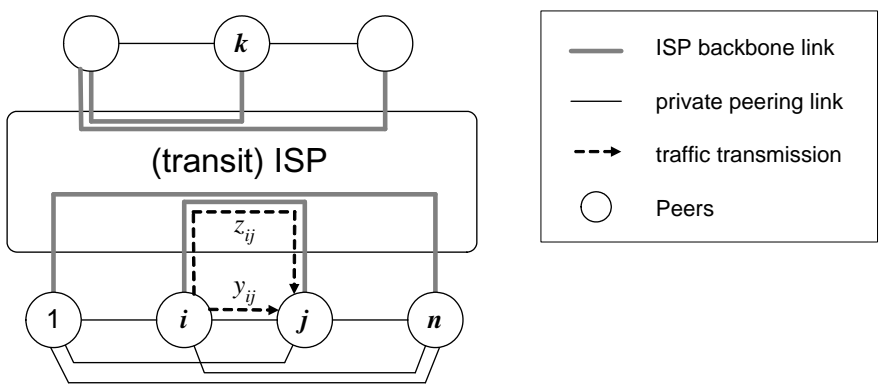

Fig. 2. A model of $n$ peers and one ISP. Each peer has one aggregate link to the ISP and possibly $n-1$ private links to other peers. Peer $i$ can communicate with peer $j$ in two possible ways: one through the peering link $l_{i j}$ and one through the ISP link $l_{i i}$. The traffic rate on link $l_{i j}$ is $y_{i j}$ while the traffic rate on link $l_{i i}$ is $z_{i j}$.

Consider a network which is depicted in Figure 2. For clarity of presentation, Table I also lists all notations used in our mathematical model. The network consists of $n$ peers and one Internet Service Provider (ISP), where a peer can be viewed as a local ISP ${ }^{1}$ and the higher level ISP is to provide connection between these peers. Peers need to communicate with each other by sending data. They can communicate with each other either by sending traffic through the ISP, or by the private peering links between themselves. In order to provide connectivity, the ISP has a communication network (in which we abstract it as a link) that has a total capacity of $n \mathcal{C}$ (in units of bps). For each peer $i \in\{1,2, \ldots, n\}$, it possesses an aggregate link to the ISP and possibly $n-1$ private peering links connecting to the other

\footnotetext{
${ }^{1}$ Unless we state otherwise, we use the term peer to denote a local ISP while the term ISP to denote higher level ISP, such as tier-1 ISP.
} 


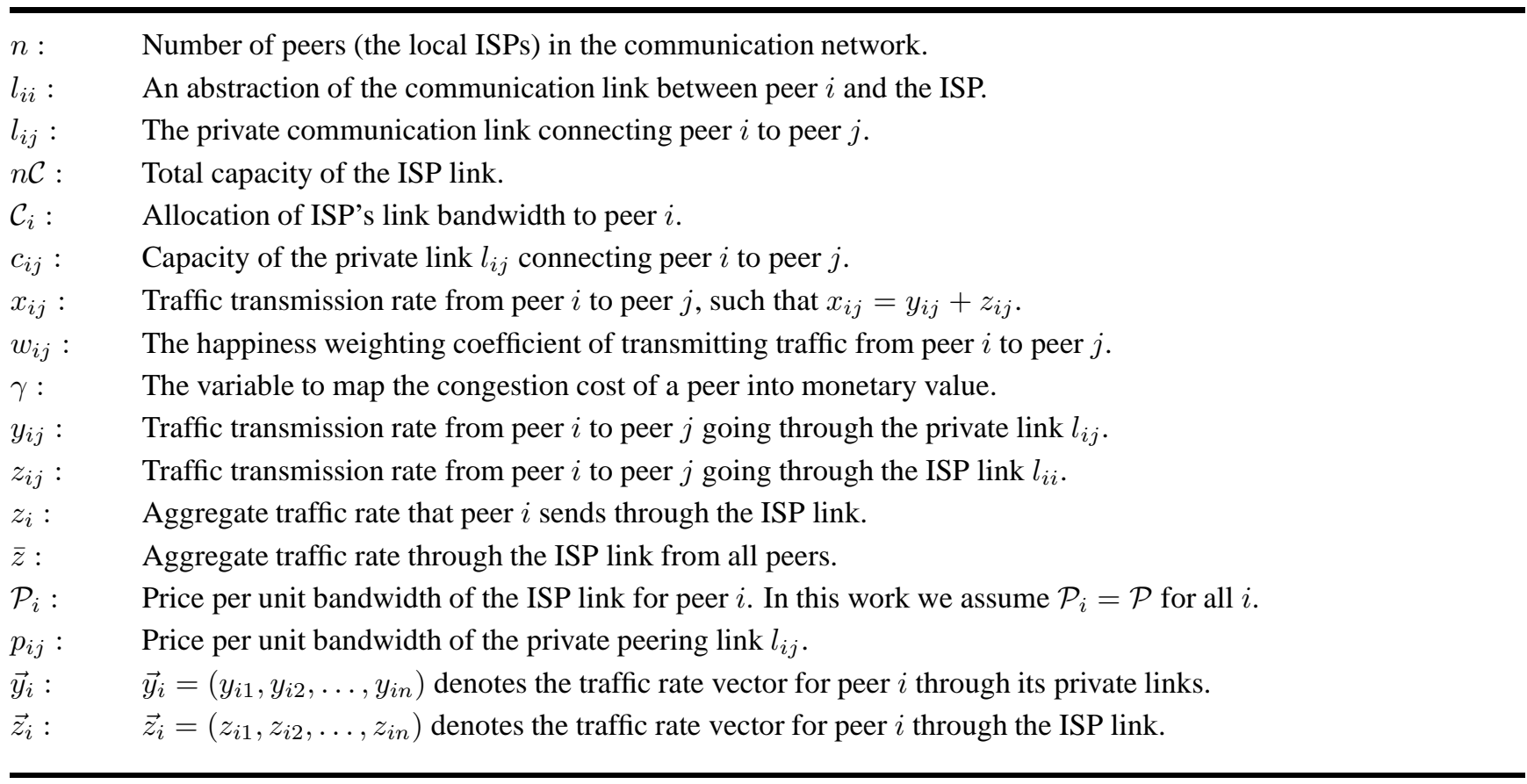

\section{TABLE I}

NOTATIONS USED TO REPRESENT THE COMMUNICATION NETWORK BETWEEN PEERS AND THE ISP

$n-1$ peers. Since the peering links are privately owned infrastructures by two parties, we use the terms "private links" and "peering links" interchangeably in the rest of the paper. Let $l_{i j}$ denote the peering link between peer $i$ and peer $j$ and this link has a capacity of $c_{i j}$ (in unit of bps). Note that if we set $c_{i j}=0$, it implies that there is no peering link between peer $i$ and peer $j$. The link connecting peer $i$ and the ISP is denoted as $l_{i i}$, and the ISP allocates $\mathcal{C}_{i}$ amount of bandwidth (in units of bps) for this connection. Note that our model can be viewed as a generalization of the network model in [2], in which private peering links are not considered.

Let $x_{i j}$ denote the transmission rate (in unit of bps) from peer $i$ to peer $j$. In short, it is the traffic originated from peer $i$ destined to peer $j$. To sustain the transmission rate of $x_{i j}$, peer $i$ obtains a utility of $A_{i j}\left(x_{i j}\right)$ where $A_{i j}$ is a strictly concave function in $x_{i j}$. As noted in [8], concave function is commonly used to represent elastic traffic, which is the dominant traffic in the Internet. The utility $A_{i j}\left(x_{i j}\right)$ represents the happiness of peer $i$ by sending data to peer $j$ at a rate of $x_{i j}$. In this paper, we use a weighted $\log$ function as our utility function and $A_{i j}\left(x_{i j}\right)=w_{i j} \log \left(1+x_{i j}\right)$. The weighting $w_{i j}$ can be interpreted as the happiness weighting coefficient of transmitting traffic between peer $i$ and peer $j$. Therefore, it is possible for $w_{i j}>w_{i k}$, which represents that peer $i$ prefers to communicate with peer $j$ than peer $k$. Note that the log function is chosen as it leads to a proportionally fair resource allocation if proper congestion control is used. Additionally, this type of utility function is also commonly used for 
performing distributed admission control[2].

The traffic transmission rate $x_{i j}$, which has to be computed later, can either go through the ISP link $l_{i i}$, or the private link $l_{i j}$. We denote $y_{i j}$ as the traffic rate that peer $i$ decides to transmit through the private link $l_{i j}$, and $z_{i j}$ as the traffic rate through ISP link $l_{i i}$. In other words, the traffic transmission rate $x_{i j}$ is equal to

$$
x_{i j}=y_{i j}+z_{i j} \quad \text { for } i, j \in\{1, \ldots, n\} .
$$

A particular case to note is the traffic rate $x_{i i}$, which denotes the traffic rate from peer $i$ to destinations other than the $n-1$ peers. This type of traffic can represent data to other part of the Internet wherein peer $i$ has to send the data through the ISP. Since there is no established private link to those outsiders, peer $i$ can only rely on the ISP link for the traffic transmission. Therefore,

$$
y_{i i}=0 \text { and } x_{i i}=z_{i i} \text { for } i \in\{1, \ldots, n\} .
$$

For the ease of presentation, let $z_{i}=\sum_{j=1}^{n} z_{i j}$ denote the aggregate traffic rate that peer $i$ sends through the ISP link, and let $\bar{z}=\sum_{j=1}^{n} z_{j}$ denote the aggregate traffic on the ISP link from all $n$ peers.

To transmit data across the ISP, peers need to pay the network operators for the transmission service. The price per unit bandwidth through the ISP link $l_{i i}$ is $\mathcal{P}_{i}$, which is determined by the ISP. Peer $i$ can also send the traffic $y_{i j}$ through the private link $l_{i j}$, and the price per unit bandwidth is $p_{i j}$, which can be mutually agreed upon between peers $i$ and $j$. In this work, we do not consider the issues on the cost of setting up peering links, since it is not part of the operating cost. We assume peers can utilize existing peer links with fixed capacities $c_{i j}$ 's. For convenience, we denote $\vec{y}_{i}=\left(y_{i 1}, y_{i 2}, \ldots, y_{i n}\right)$ as the traffic rate vector for peer $i$ through its private links and $\vec{z}_{i}=\left(z_{i 1}, z_{i 2}, \ldots, z_{i n}\right)$ as the traffic rate vector for peer $i$ through the ISP link. We denote $\overrightarrow{\mathcal{P}}=\left(\mathcal{P}_{1}, \mathcal{P}_{2}, \ldots, \mathcal{P}_{n}\right)$ as the vector of ISP prices set on different peers.

Besides paying the ISP for the transmission service, each peer also needs to take into consideration of the congestion costs on the links. If we assume that all links can be represented by an $\mathrm{M} / \mathrm{M} / 1$ model as in [2], one can take the delay on the link as its congestion indication or cost. Rather than informing all peers about the current transmission rate $\bar{z}$ on the ISP link (which can be considered as confidential information by a peer), ISP will do a pre-computation of our propose algorithm and announce its bandwidth allocation to peer $i$ as $\mathcal{C}_{i}$. There is also a technical merit for this announcement which will be discussed in detail in later sections. Under this form of setting, the congestion cost $D_{i j}$ of a link $l_{i j}$, is

$$
D_{i j}= \begin{cases}\frac{1}{c_{i j}-y_{i j}} & \text { if } i \neq j \\ \frac{1}{\mathcal{C}_{i}-z_{i}} & \text { if } i=j\end{cases}
$$


To model the economic incentives and behaviors of all peers, we consider the following optimization. The objective of peer $i$ is to maximize the following function:

$$
\begin{aligned}
& \operatorname{Max} U_{i}=\sum_{j} w_{i j} \log \left(1+y_{i j}+z_{i j}\right)-\mathbf{1}_{\left\{z_{i} \neq 0\right\}}\left[\frac{\gamma}{\mathcal{C}_{i}-z_{i}}\right]-\mathcal{P}_{i} z_{i}-\sum_{j \neq i} 1_{\left\{y_{i j} \neq 0\right\}}\left[\frac{\gamma}{c_{i j}-y_{i j}}\right]-\sum_{j \neq i} p_{i j} y_{i j} \\
& \text { s. t. } \quad 0 \leq y_{i j} \leq c_{i j} \text { for all } j \neq i, \quad y_{i i}=0, \quad \sum_{j} z_{i j} \leq \mathcal{C}_{i}, \quad z_{i j} \geq 0 \text { for all } j .
\end{aligned}
$$

where $\mathbf{1}_{\{p\}}$ is an indicator function which equals to 1 if the condition $p$ is true, or 0 otherwise. The objective function of Equation (1) represents the economic incentive for peer $i$ to perform traffic transmission. In here, $w_{i j} \log \left(1+y_{i j}+z_{i j}\right)$ is the happiness of peer $i$ by sending traffic to peer $j$. The term $\frac{\gamma}{\mathcal{C}_{i}-z_{i}}$ is the congestion cost of peer $i$ on the ISP link. The variable $\gamma>0$, indicates the impact of congestion cost to a peer and it converts the congestion cost of a peer into monetary value. The larger the value of $\gamma$, the more the peers' concern is on the congestion cost. In later section, we also show the simulation results indicating the impact of $\gamma$ on the convergence point of the traffic transmission rates. However, if peer $i$ does not transmit through the ISP link, it does not bear the delay load and the congestion cost will be zero. The term $\mathcal{P}_{i} z_{i}$ is the total payment of peer $i$ to the ISP. Similarly, $\frac{\gamma}{c_{i j}-y_{i j}}$ is the congestion cost on the peering link connecting peer $i$ to peer $j$, when the transmission rate on $l_{i j}$ is non-zero. Lastly, peer $i$ has a payment of $p_{i j} y_{i j}$ to peer $j$ for using the private link ${ }^{2}$. Now, the happiness, congestion cost and payment are mapped to the same monetary domain with $w_{i j}$ and $\gamma$. If the parameters, $w_{i j}, \gamma, \mathcal{C}_{i}, \mathcal{P}_{i}, c_{i j}, p_{i j}$ are set properly, peer $i$ 's happiness, congestion cost and payment can be measured in monetary units.

Meanwhile, constraints represented in Equation (2) define the feasible region of this optimization problem. The first are the non-negative and capacity constraints of the peering links. The second constraint is due to the absence of peering links established to the "outsiders". The third and fourth are the capacity and non-negative constraints of the ISP link respectively. In summary, each peer $i$ needs to determine the proper traffic rates vectors $\vec{z}_{i}$ and $\vec{y}_{i}$ so as to maximize its aggregate utility in Equation (1).

Note that the optimization processes of different peers are not independent. For each peer $i$, given the bandwidth allocation $\mathcal{C}_{i}$ of the ISP link, it performs an optimization and determines its optimal transmission rate $z_{i}$ and bids to the ISP. After collecting the bidding information from all peers, the ISP will calculate the new bandwidth allocation according to the new biddings, as well as different resource allocation criteria (which is to be discussed in Section V). Therefore, the interaction process between peers can be modelled as a non-cooperative game such that each peer offers a bid to maximize its own utility.

Under this framework, for a given ISP price vector $\overrightarrow{\mathcal{P}}=\left(\mathcal{P}_{1}, \mathcal{P}_{2}, \ldots, \mathcal{P}_{n}\right)$, this defines a non-cooperative

${ }^{2}$ It is also possible for us to model the case that peer $i$ and $j$ do not charge each other for sending peering traffic, i.e., by setting $p_{i j}=p_{j i}=0$. 
game between these $n$ peers [15]. They interact with each other and determine their optimal transmission rates periodically and asynchronously. Given the existence of an equilibrium point, the operating point for $n$ peers is the solution to the Nash equilibrium of this game. For each price vector $\overrightarrow{\mathcal{P}}>0$, a Nash equilibrium point for this $n$-peers game is defined as two $n$-tuples $y^{*}=\left(\vec{y}_{1}^{*}, \vec{y}_{2}^{*}, \ldots, \vec{y}_{n}^{*}\right)$ and $z^{*}=\left(\vec{z}_{1}^{*}, \vec{z}_{2}^{*}, \ldots, \vec{z}_{n}^{*}\right)$, such that for all peers $i \in\{1,2, \ldots, n\}$ :

$$
U_{i}\left(y^{*}, z^{*}, \overrightarrow{\mathcal{P}}\right) \geq U_{i}(y, z, \overrightarrow{\mathcal{P}})
$$

for any other feasible traffic vector $y=\left(\vec{y}_{1}, \vec{y}_{2}, \ldots, \vec{y}_{n}\right)$ and $z=\left(\vec{z}_{1}, \vec{z}_{2}, \ldots, \vec{z}_{n}\right)$ that satisfies the constraints defined in Equation (2).

On the other hand, the ISP is associated with a revenue maximization problem:

$$
\text { Maximize } \mathcal{P} \cdot \bar{z}^{*}(\mathcal{P}) \quad \text { over } \mathcal{P} \geq 0
$$

where $\bar{z}^{*}(\mathcal{P})=\sum_{j} z_{j}^{*}(\mathcal{P})$ is the aggregate traffic on ISP link at the Nash equilibrium. In here, note that we assume the ISP charges the same price for all peers and there is no price discriminate. Therefore $\mathcal{P}_{i}=\mathcal{P}$ for all $i$. This equivalently defines a Stackelberg game [15] with one leader (ISP) and the noncooperative Nash followers ( $n$ peers). The ISP has a first move advantage to determine the optimal price such that its own revenue can be maximized.

\section{Maximization by Individual Peers with traffic demand is a constant}

The traffic demand $x_{i j}$ can be viewed as an aggregate request from the customers of peer $i$ destining to peer $j$ and is a constant within an operating period. Consider also the case when peer $i$ can always obtain a sufficient bandwidth capacity to transmit all the aggregate requests, i.e. $\sum_{j} x_{i j} \leq \sum_{j} c_{i j}+\mathcal{C}_{i}$, then the peer will transmit all the requests, while maximizing its utility at the same time. With fixed traffic demands $x_{i j}$ 's, the aggregate happiness $\sum_{j} A_{i j}\left(x_{i j}\right)$ is therefore a constant. The objective function of peer $i$ can then be transformed as to minimize the aggregate congestion costs and payments. Substitute $y_{i j}=x_{i j}-z_{i j}$ in Equation (1), we have the following transformed optimization:

$$
\begin{aligned}
\operatorname{Max} U_{i}= & K-\sum_{j \neq i} \mathbf{1}_{\left\{z_{i j} \neq x_{i j}\right\}}\left[\frac{\gamma}{c_{i j}-x_{i j}+z_{i j}}\right]+\sum_{j \neq i} p_{i j} z_{i j}-\mathbf{1}_{\left\{z_{i} \neq 0\right\}}\left[\frac{\gamma}{\mathcal{C}_{i}-z_{i}}\right]-\mathcal{P}_{i} z_{i} \\
\operatorname{Min} \mathcal{V}_{i}= & \sum_{j \neq i} \mathbf{1}_{\left\{z_{i j} \neq x_{i j}\right\}}\left[\frac{\gamma}{c_{i j}-x_{i j}+z_{i j}}\right]-\sum_{j \neq i} p_{i j} z_{i j}+\mathbf{1}_{\left\{z_{i} \neq 0\right\}}\left[\frac{\gamma}{\mathcal{C}_{i}-z_{i}}\right]+\mathcal{P}_{i} z_{i} \\
\text { s. t. } & \max \left\{0, x_{i j}-c_{i j}\right\} \leq z_{i j} \leq x_{i j} \quad \text { for all } j \neq i, \quad z_{i i}=x_{i i}, \quad \sum_{j} z_{i j} \leq \mathcal{C}_{i}
\end{aligned}
$$

where $K=\sum_{j} A_{i j}\left(x_{i j}\right)-\sum_{j \neq i} p_{i j} x_{i j}$ is a constant. 
The objective of the new optimization problem (5) is to minimize the aggregate congestion costs and payments under constant traffic demands. The variable transmission rate vector $\vec{y}_{i}$ is absorbed and the remaining variable in the new optimization problem is $\vec{z}_{i}$. The constraints in Equation (6) represent the feasible region of the ISP link transmission rates. The first constraints give the lower and upper bounds for $z_{i j}$ 's. When $c_{i j} \geq x_{i j}$, the bandwidth in the private peering link $l_{i j}$ is larger than the demand $x_{i j}$, i.e. private peering link capacity is sufficient for the demand and so the minimum transmission rate in ISP link $z_{i j}$ is zero. When $c_{i j}<x_{i j}$, the bandwidth in the private peering link is insufficient for the demand and so part of the traffic must go through the ISP link. It makes the minimum value of $z_{i j}=x_{i j}-c_{i j}$. The second constraint again is due to the absence of private peering link to the "outsiders". The third constraint is the ISP link capacity constraint.

\section{A. Distributed Solution of the Minimization Problem}

In the following, we illustrate how a peer, say $i$, can determine its transmission rates, that is $\vec{z}_{i}$, rates to other peers via the ISP's link, as well as $\vec{y}_{i}$, rates to other peers via private peering links, so as to minimize its cost when the bandwidth supply is sufficient. Assuming that the peer knows the price $\mathcal{P}_{i}$ specified by the ISP and the associated bandwidth allocation $\mathcal{C}_{i}$, one can model an individual peer's behavior as a convex optimization problem as defined in Equation (5). Let us first study the necessary and boundary conditions for a peer to minimize the cost.

\section{Necessary conditions with positive transmission rate}

Since the $\operatorname{cost} \mathcal{V}_{i}$ is discontinuous at $z_{i j}=x_{i j}$ (i.e., transmission rate through the private peering link $l_{i j}$ is zero) and $z_{i}=0$ (i.e., transmission rate through the ISP link is zero), we first investigate the necessary conditions when $z_{i j} \neq x_{i j}$ and $z_{i} \neq 0$. The optimization problem of Equation (5) has $n-1$ variables (with $z_{i i}=x_{i i}$ ). The first and second order partial derivatives with respect to $z_{i j}$ and $z_{i k}$ for $k \neq j \neq i$ are:

$\frac{\partial \mathcal{V}_{i}}{\partial z_{i j}}=\frac{-\gamma}{\left(c_{i j}-x_{i j}+z_{i j}\right)^{2}}-p_{i j}+\frac{\gamma}{\left(\mathcal{C}_{i}-z_{i}\right)^{2}}+\mathcal{P}_{i}, \frac{\partial^{2} \mathcal{V}_{i}}{\partial z_{i j}^{2}}=\frac{2 \gamma}{\left(c_{i j}-x_{i j}+z_{i j}\right)^{3}}+\frac{2 \gamma}{\left(\mathcal{C}_{i}-z_{i}\right)^{3}}>0, \quad \frac{\partial^{2} \mathcal{V}_{i}}{\partial z_{i j} \partial z_{i k}}=\frac{2 \gamma}{\left(\mathcal{C}_{i}-z_{i}\right)^{3}}>0$

This shows that the Hessian matrix of the objective function in Equation (5) is positive definite on the non-negative space bounded by the capacity constraints $x_{i j}-c_{i j} \leq z_{i j} \leq x_{i j}$ and $z_{i} \leq \mathcal{C}_{i}$. So the cost $\mathcal{V}_{i}$ is strictly convex in $z_{i j}$ for all $j \neq i$. The minimum cost and optimizer to this problem is unique and can be found by the Lagrangian method. The necessary conditions of $z_{i j}$ for the minimization of $\mathcal{V}_{i}$ are:

$$
\frac{\partial \mathcal{V}_{i}}{\partial z_{i j}} \quad \begin{cases}>0 & \text { if } z_{i j}=0 \\ =0 & \text { if } z_{i j}>0\end{cases}
$$




\section{Boundary cases to Maximization problem}

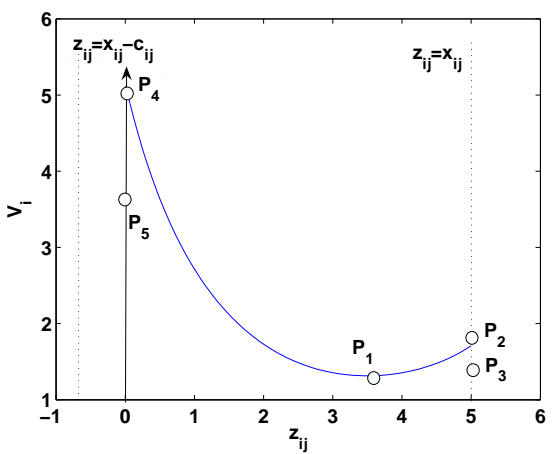

(a)

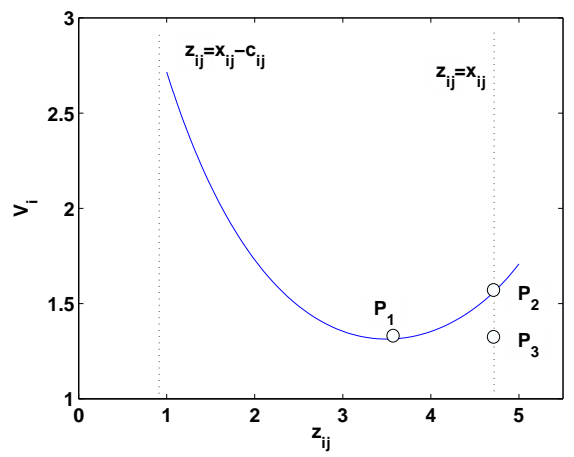

(b)

Fig. 3. Peer $i$ 's cost against transmission rate $z_{i j}$ when $z_{i j}^{*}=\arg \left\{\frac{\partial \mathcal{V}_{i}}{\partial z_{i j}}=0\right\}$ is in the feasible range: (a) when $x_{i j} \leq c_{i j}$, (b) when $x_{i j}>c_{i j}$.

Due to the discontinuity of the objective function, the necessary conditions given above may not achieve the global minimum. In here we explore the boundary cases when the transmission rates are zero, i.e., $z_{i j}=x_{i j}$ or $z_{i}=0$. Figures 3 and 4 show these cases. Figure 3 corresponds to the case when $z_{i j}^{*}=\arg \left\{\frac{\partial \mathcal{V}_{i}}{\partial z_{i j}}=0\right\}$ is in the feasible range. The vertical axis shows the aggregate cost $\mathcal{V}_{i}$ and the horizontal axis shows the transmission rate $z_{i j}$. Figure 3(a) considers if $x_{i j} \leq c_{i j}$, which implies the private peering link capacity is adequate for the transmission demand; and Figure 3(b) considers if $x_{i j}>c_{i j}$, which implies the private peering link capacity is inadequate for the transmission demand. In Figure 3, the minimum point of the curve is at $P 1$ when $z_{i j}=z_{i j}^{*}$. We first consider the upper bound. When $z_{i j}=x_{i j}$, the transmission rate goes through the ISP link only. The congestion cost in peering link $l_{i j}$ is not considered and is subtracted from $\mathcal{V}_{i}$, so $P 3$ rather than $P 2$ is the point of $\mathcal{V}_{i}$ when $z_{i j}=x_{i j}$. We then consider the lower bound under two cases: case i) when $x_{i j} \leq c_{i j}$ (as in Figure 3(a)), the minimum value of $z_{i j}=0$. If there is a $z_{i k}>0$ for some $k \neq j, P 4$ is the point when $z_{i j}=0$. But if the aggregate traffic through the ISP link is zero $\left(z_{i}=0\right), P 5$ is the point when $z_{i j}=0$. Note that the congestion cost in the ISP link is subtracted from $\mathcal{V}_{i}$ in this case; case ii) when $x_{i j}>c_{i j}$ (as in Figure 3(b)), the minimum value of $z_{i j}=x_{i j}-c_{i j}$. This is because the maximum amount of traffic through the private peering link is $r_{i j}$, the remaining rate $x_{i j}-c_{i j}$ has to go through the ISP link and the congestion cost in the ISP link must be considered. In general, when $z_{i j}^{*}$ is in the feasible range, the optimal transmission rate is either $z_{i j}=0, z_{i j}=x_{i j}$ or $z_{i j}=z_{i j}^{*}$.

Figure 4 illustrates when $z_{i j}^{*}=\arg \left\{\frac{\partial \mathcal{V}_{i}}{\partial z_{i j}}=0\right\}$ is not in the feasible range. Figure 4(a) considers when 


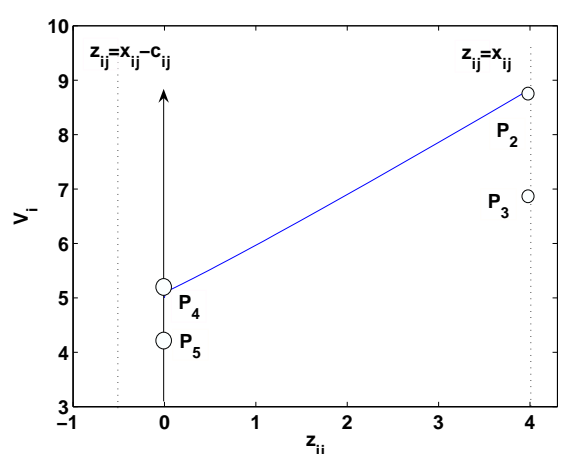

(a)

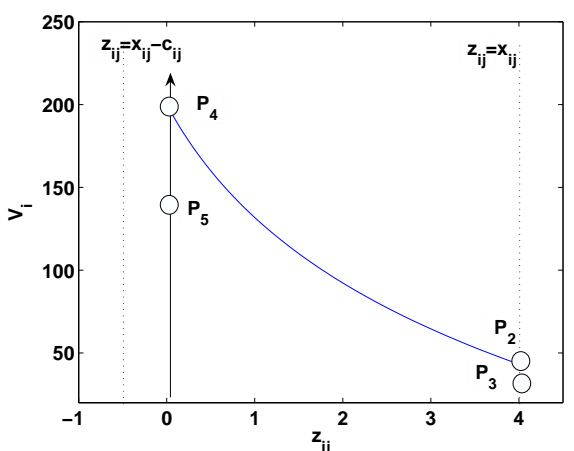

(b)

Fig. 4. Peer $i$ 's cost against transmission rate $z_{i j}$ when $z_{i j}^{*}=\arg \left\{\frac{\partial \mathcal{V}_{i}}{\partial z_{i j}}=0\right\}$ is not in the feasible range: (a) when $z_{i j}^{*} \leq \min \left\{0,\left(x_{i j}-c_{i j}\right)\right\},(\mathrm{b})$ when $z_{i j}^{*} \geq x_{i j}$.

$z_{i j}^{*} \leq \min \left\{0,\left(x_{i j}-c_{i j}\right)\right\}$. As $\mathcal{V}_{i}$ is strictly convex in $z_{i j}$, the minimum feasible $z_{i j}=\min \left\{0,\left(x_{i j}-c_{i j}\right)\right\}$, is either at $P 4$ (when $z_{i}>0$ ) or at $P 5$ (when $z_{i}=0$ ). For the upper bound of $z_{i j}$, when $z_{i j}=x_{i j}$, the congestion cost in the private peering link is subtracted from the cost. This concludes that the minimum point of $\mathcal{V}_{i}$ is either when $z_{i j}=\min \left\{0,\left(x_{i j}-c_{i j}\right)\right\}$ (optimizer is either $P 4$ or $P 5$ ) or when $z_{i j}=x_{i j}$ (optimizer is P3). Figure 4(b) shows the case when $z_{i j}^{*}>x_{i j}$. The maximum feasible $z_{i j}=x_{i j}$ due to the convexity of $\mathcal{V}_{i}$. For the lower bound of $z_{i j}$, when $z_{i}=0$ (which implies $z_{i j}=0$ ), the value of $\mathcal{V}_{i}$ at $P 5$ may be smaller than that at $P 3$. This concludes that the minimum point of $\mathcal{V}_{i}$ is either $P 3$ when $z_{i j}=x_{i j}$ or $P 5$ when $z_{i j}=0$. Lastly, after the ISP link transmission rates $z_{i j}$ 's are computed, the private peering link transmission rates $y_{i j}$ 's can be found by $y_{i j}=x_{i j}-z_{i j}$.

\section{Solution to the general case of Maximization Problem by Individual Peers}

In this section, we illustrate how a peer, say $i$, can determine its transmission rates, which is $\vec{z}_{i}$, to other peers via the ISP, as well as the transmission rate $\vec{y}_{i}$, rates to other peers via peering links, so as to maximize its utility. Assuming that the peer knows the price $\mathcal{P}$ specified by the ISP and the associated bandwidth allocation $\mathcal{C}_{i}$, one can model an individual peer's behavior as a convex optimization problem as defined in Equation (1). In this section, we investigate the necessary and boundary conditions for a peer to maximize its utility.

\section{A. Necessary conditions with positive transmission rate}

Since $U_{i}$ is discontinuous at $y_{i j}=0$ (i.e., the traffic rate through the peering link $l_{i j}$ is zero) and $z_{i}=0$ (i.e., the traffic rate through the ISP's link $l_{i i}$ is zero), we first investigate the necessary conditions when $y_{i j} \neq 0$ and $z_{i} \neq 0$. The optimization problem of Equation (1) has $2 n-1$ variables (with $y_{i i}=0$ ). We 
first write down the second order partial derivatives with respect to $y_{i j}$ and $z_{i j}$ :

$\frac{\partial^{2} U_{i}}{\partial y_{i j}^{2}}=\frac{-w_{i j}}{\left(1+y_{i j}+z_{i j}\right)^{2}}-\frac{2 \gamma}{\left(c_{i j}-y_{i j}\right)^{3}}<0, \frac{\partial^{2} U_{i}}{\partial z_{i j}^{2}}=\frac{-w_{i j}}{\left(1+y_{i j}+z_{i j}\right)^{2}}-\frac{2 \gamma}{\left(\mathcal{C}_{i}-z_{i}\right)^{3}}<0, \frac{\partial^{2} U_{i}}{\partial y_{i j} \partial z_{i j}}=\frac{-w_{i j}}{\left(1+y_{i j}+z_{i j}\right)^{2}}<0$.

And for $k \neq i \neq j$, the second order partial derivatives of Equation (1) with respect to $y_{i k}$ and $z_{i k}$ are:

$$
\frac{\partial^{2} U_{i}}{\partial y_{i j} \partial y_{i k}}=0, \quad \frac{\partial^{2} U_{i}}{\partial y_{i j} \partial z_{i k}}=0, \quad \frac{\partial^{2} U_{i}}{\partial z_{i j} \partial z_{i k}}=\frac{-2 \gamma}{\left(\mathcal{C}_{i}-z_{i}\right)^{3}}<0
$$

Therefore, the Hessian matrix [1] of the objective function in Equation (1) is negative definite on the non-negative value bounded by $y_{i j} \leq c_{i j}$ and $z_{i} \leq \mathcal{C}_{i}$. So $U_{i}$ is strictly concave in $y_{i j}$ and $z_{i j}$ for all $j$. The maximum utility and optimizer to this problem is unique and can be found by the Lagrangian method [16]. The necessary conditions of $y_{i j}$ and $z_{i j}$ for the maximization of $U_{i}$ are:

$$
\frac{\partial U_{i}}{\partial y_{i j}}\left\{\begin{array}{ll}
<0 & \text { if } y_{i j}=0 \\
=0 & \text { if } y_{i j}>0
\end{array}, \quad \frac{\partial U_{i}}{\partial z_{i j}} \begin{cases}<0 & \text { if } z_{i j}=0 \\
=0 & \text { if } z_{i j}>0 .\end{cases}\right.
$$

\section{B. Boundary cases}

Due to the discontinuity of the objective function, the necessary conditions given above may not achieve the global maximum. In here, we are going to explore the boundary cases when the transmission rate tends to be zero, i.e., $y_{i j}=0$ or $z_{i}=0$. Figure 5 shows the illustration of an example. We plot the utility of peer $i$ against one particular variable $y_{i j}\left(z_{i j}\right.$ is similar). Figure 5(a) corresponds to the case when $\left.\frac{\partial U_{i}}{\partial y_{i j}}\right|_{y_{i j}=0}<0$. The optimizer is $y_{i j}=0$, but the maximum utility is at point $P_{1}$ rather than point $P_{2}$ since there is no congestion cost at the private link when $y_{i j}=0$. Figure 5(b) corresponds to the case when $y_{i j}^{*}=\arg \left\{\frac{\partial U_{i}}{\partial y_{i j}}=0\right\}$ is positive ( $P_{3}$ in the figure). If the utility $U_{i}$ at the boundary point $P_{2}$ which is less than $P_{3}, P_{3}$ is the maximum point and $y_{i j}^{*}$ is the optimizer. However, there exists a case when the utility $U_{i}$ at the boundary point $P_{1}$ is greater than that of point $P_{3}$. Therefore, $P_{1}$ should be the maximum point and $y_{i j}=0$ is the optimizer.

Here we provide the physical interpretation of the two cases illustrated in Figure 5(b). If the utility $U_{i}$ at the boundary point is $P_{2}$, it indicates that when the transmission rate $y_{i j}$ increases, the increase in happiness outweighs the increases in congestion cost and its total payment, thus achieving the maximum utility at point $P_{3}$. However, if the utility $U_{i}$ at the boundary point is $P_{1}$, it means that when the transmission rate increases, the increase in happiness cannot compensate for the increases in congestion cost and its payments. That is, although peer $i$ achieves the maximum utility at $P_{3}$, the utility is negative. So the best strategy for peer $i$ is not to transmit data through $l_{i j}$ at all. Note that when a peer $i$ does not send through any links, it gets a zero happiness, zero congestion cost and zero payment, and thus a zero 
utility. Therefore, a peer will always achieve a non-negative utility, since in the worse case, it can opt not to transmit and leave the network (or market).

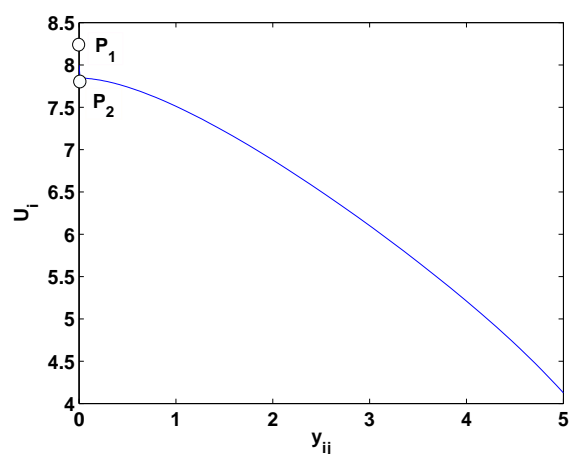

(a)

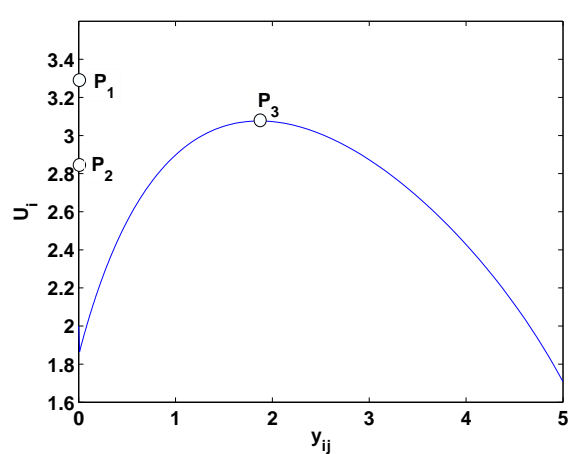

(b)

Fig. 5. Utility of peer $i$ against one particular $y_{i j}$ (a) negative optimizer (b) positive optimizer.

\section{Example of a Peer's Strategy}

Let us end this section with a simple example to illustrate the peer's optimization strategy. There are three peers in the network. Each peer has two private links connecting to other peers with a homogeneous capacity $c_{i j}=5$ and the same unit price $p_{i j}=1$. The happiness weighting coefficients for peer 1 are $w_{11}=10, w_{12}=5$ and $w_{13}=1$ respectively. The mapping variable $\gamma=1$. ISP allocates a capacity $\mathcal{C}_{1}=20$ to peer 1 . The unit price for the ISP link is $\mathcal{P}=1$.

To find out the optimal rate vectors of peer 1 , we list out the necessary conditions with positive transmission rate:

$$
\begin{aligned}
& \frac{5}{1+y_{12}+z_{12}}-\frac{1}{\left(5-y_{12}\right)^{2}}-1 \begin{cases}<0 & \text { if } y_{12}=0 \\
=0 & \text { if } y_{12}>0\end{cases} \\
& \frac{1}{1+y_{13}+z_{13}}-\frac{1}{\left(5-y_{13}\right)^{2}}-1 \begin{cases}<0 & \text { if } y_{13}=0 \\
=0 & \text { if } y_{13}>0\end{cases} \\
& \frac{10}{1+z_{11}}-\frac{1}{\left(20-z_{1}\right)^{2}}-1 \begin{cases}<0 & \text { if } z_{11}=0 \\
=0 & \text { if } z_{11}>0\end{cases} \\
& \frac{5}{1+y_{12}+z_{12}}-\frac{1}{\left(20-z_{1}\right)^{2}}-1 \begin{cases}<0 & \text { if } z_{12}=0 \\
=0 & \text { if } z_{12}>0\end{cases} \\
& \frac{1}{1+y_{13}+z_{13}}-\frac{1}{\left(20-z_{1}\right)^{2}}-1 \begin{cases}<0 & \text { if } z_{13}=0 \\
=0 & \text { if } z_{13}>0\end{cases}
\end{aligned}
$$

Solving the system of equations gives the optimal rate vectors of $\vec{y}_{1}=\{0,0\}$ and $\vec{z}_{1}=\{8.8,3.9,0\}$ and 
utility $U_{1}=20.07$. Finally we need to compare this with the utility achieved at the boundary points as elaborated in Figure 5(b), to make sure that the rate vectors really achieve the global optimal.

\section{Distributed Resource Allocation by ISP}

From an ISP's point of view, a monopolized use of its link bandwidth surely reduces its customer size and so increases the risk of the business. Moreover, in order to maximize its revenue, an ISP has to know approximately the demand of its link bandwidth. Therefore, an ISP wants to have an efficient resource allocation algorithm. Now, given the total amount of resource $n \mathcal{C}$ (ISP's link bandwidth), the ISP needs to determine how to distribute this common resource to all the $n$ peers. In this section, we propose two different resource allocation algorithms that can be adopted by the ISP.

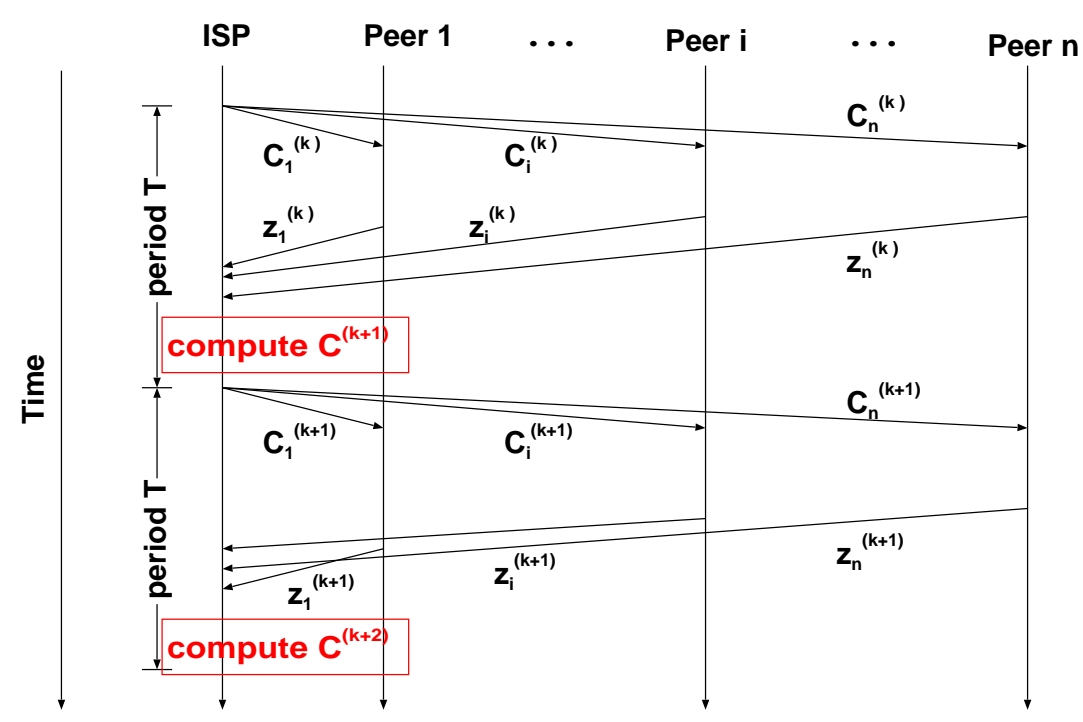

Fig. 6. A general framework for the ISP to obtain bidding information from peers and distribute resulting information back to peers.

Before we proceed to the formal presentation of the algorithms, let us illustrate the general framework under which the ISP can interact with peers so that the ISP is able to discover the actual resource demands from peers, and also peers are informed about the pricing information and the available resources. Figure 6 illustrates the general framework. Initially, the ISP equally distributes its capacity to all peers at time $t=0$. Each peer $i$ calculates its optimal traffic transmission rates based on the currently allocated ISP capacity $\mathcal{C}_{i}$, as well as the ISP link price $\mathcal{P}$. Then the peer reports its transmission rate (resource usage), $z_{i}$, back to the ISP. We refer to the feedback information $z_{i}$ as the bidding of peer $i$. The ISP receives the biddings from peers within a period of time $T$. At the end of each period, ISP recomputes the link resource distribution and sends the new bandwidth allocation $\mathcal{C}_{i}$ to peer $i$, where $i \in\{1, \ldots, n\}$. Based on the new bandwidth allocation, peers calculate their optimal transmission rates again and the process repeats.

There are two advantages for this framework. First, all the information that a peer $i$ requires are the 
unit prices $p_{i j}$ 's and capacities $c_{i j}$ 's of its private links and the allocated link capacity $\mathcal{C}_{i}$, as well as the informed price $\mathcal{P}$. These can be seen as the private information of peer $i$. Peer $i$ does not have to know the bandwidth allocation $\left\{\mathcal{C}_{1}, \ldots, \mathcal{C}_{n}\right\}$ and transmission rates $\left\{z_{1}, \ldots, z_{n}\right\}$ of all other peers, which is considered as confidential information. On the other hand, when the ISP makes the bandwidth allocation, what it requires to know are the biddings $\left(z_{1}, z_{2}, \ldots, z_{n}\right)$ from all peers. The ISP may not know the utility functions and the information about private links of these peers (i.e., $p_{i j}$ and $c_{i j}$ for all $i, j$ ). Secondly, the overhead of information exchange in this framework is small. ISP only needs to inform each peer its allocated capacity, while each peer only needs to reply to the ISP its bidding.

In the following, we present two resource allocation algorithms by which the ISP can determine the appropriate capacity $C_{i}$ for all peers $i, i=1, \cdots, n$.

\section{A. Proportional Share Algorithm (PSA)}

Under the Proportional Share Algorithm, ISP distributes its remaining capacity proportionally to the biddings of peers received within each period. Initially, ISP distributes its capacity equally to every peer, i.e., $\mathcal{C}_{i}=\frac{n \mathcal{C}}{n}=\mathcal{C}$ for all $i$. The ISP sends this information to every peer. Upon receiving the information from the ISP, each peer uses the algorithm proposed in Section IV to find its optimal transfer rates (i.e., $z_{i}$ for peer $i$ ) and sends it back to the ISP as a bidding for the ISP link resource. At the end of a period, the ISP gathers all the feedback from peers. Under the PSA algorithm, ISP first allocates to each peer the amount of bandwidth equal to the peer's bidding ${ }^{3}$. And then the ISP distributes the remaining resource proportionally to the biddings of the peers. Formally, we have

$$
C_{i}=z_{i}+\frac{z_{i}}{\bar{z}}(n \mathcal{C}-\bar{z})=\frac{z_{i}}{\bar{z}} n \mathcal{C} .
$$

The algorithm of the PSA is depicted as follows:

\section{Proportional Share Algorithm:}

1. ISP initiates $C_{i}^{(0)}:=\frac{n \mathcal{C}}{n}:=\mathcal{C}$ to each peer $i$.

Set counter $k:=0$.

2. while (TRUE) \{

3. ISP passes $C_{i}^{(k)}$ to each peer $i$;

4. for (i=1 to $\mathrm{n})\{$

5. Peer $i$ computes $\vec{y}_{i}^{(k)}$ and $\vec{z}_{i}^{(k)}$ with the algorithm proposed in Section IV; and sends $z_{i}^{(k)}=\sum_{j} z_{i j}^{(k)}$ back to ISP;

6. $\} / *$ termination of for-loop */

7. $\quad$ ISP updates $C_{i}^{(k+1)}=\frac{z_{i}^{(k)}}{\bar{z}^{(k)}}(n \mathcal{C})$ for every peer $i$; update counter $\mathrm{k}:=\mathrm{k}+1$;

8. \}/* termination of while-loop */

\footnotetext{
${ }^{3} \mathrm{We}$ argue that the aggregate rate of biddings from all peers will not exceed the ISP link capacity, if each peer strictly performs the optimization problem (1). However, peers could cheat the ISP by bidding a rate higher than the allocated bandwidth $\mathcal{C}_{i}$ and receive a higher bandwidth allocation. Due to the lack of space, cheat prevention is out of scope in this paper.
} 
When the ISP link capacity is sufficiently large to support the demand from all peers, the traffic rate vectors of all peers and the resource biddings will converge quickly. However, if there is insufficient amount of resource (e.g., the happiness weighting coefficients of peers are large, while the ISP link capacity is limited) the peers with the largest happiness coefficients (i.e., $w_{i j}$ ) may be able to monopolize all the ISP capacity, leaving no capacity left for other peers. Note that even when this monopoly occurs, the ISP is still maximizing its profit under the PSA policy. However, this outcome may not be undesirable for the ISP since it may not want to have a single peer as its customer, or a small number of peers to monopolize all its resource. This drawback motivates the following algorithm.

\section{B. Equal share algorithm (ESA)}

Under the Equal Share Algorithm, ISP distributes its remaining capacity equally among all peers after satisfying their bandwidth consumption demands indicated by their biddings. Initially, ISP distributes its capacity equally to every peer, i.e., $\mathcal{C}_{i}=\mathcal{C}$ for all $i$, and sends the capacity distribution $\mathcal{C}_{i}$ to every peer $i$. Upon receiving the information from the ISP, each peer uses the algorithm proposed in Section IV to find its optimal transfer rates (i.e., $z_{i}$ for peer $i$ ) and sends the information back to the ISP as its resource bidding. Within the following period, ISP gathers all the feedbacks from peers. ISP first allocates to each peer the capacity it bids, and then the ISP distributes the remaining resource equally to the peers. Formally, we have:

$$
C_{i}=z_{i}+\frac{(n \mathcal{C}-\bar{z})}{n}
$$

The algorithm of the ESA is described as follows:

\section{Equal Share Algorithm (ESA):}

1. ISP initiates $C_{i}^{(0)}:=\frac{n \mathcal{C}}{n}:=\mathcal{C}$ to each peer $i$.

Set counter $k:=0$.

2. while (TRUE) \{

3. $\quad$ ISP passes $C_{i}^{(k)}$ to each peer $i$;

4. for ( $\mathrm{i}=1$ to $\mathrm{n})\{$

5. $\quad$ Peer $i$ computes $\vec{y}_{i}^{(k)}$ and $\vec{z}_{i}^{(k)}$ with the algorithm proposed in Section IV; and sends $z_{i}^{(k)}=\sum_{j} z_{i j}^{(k)}$ back to ISP;

6. $\} / *$ termination of for-loop * /

7. ISP updates $C_{i}^{(k+1)}=z_{i}^{(k)}+\frac{n \mathcal{C}-\bar{z}^{(k)}}{n}$ for every peer $i$; update counter $\mathrm{k}:=\mathrm{k}+1$;

8. \}/* termination of while-loop */ 


\section{Illustration of ISP Resource Allocation}

To illustrate the effectiveness and performance of the above algorithms, we carry out two experiments and illustrate the resource distribution under two different scenarios, namely, (a) the ISP has sufficient capacity, and (b) the ISP has insufficient capacity.

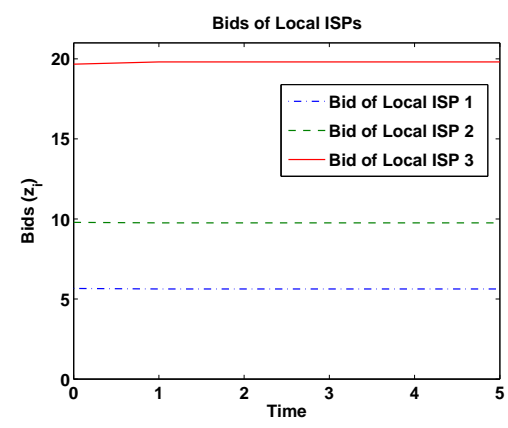

(a) PSA and $\gamma=1$

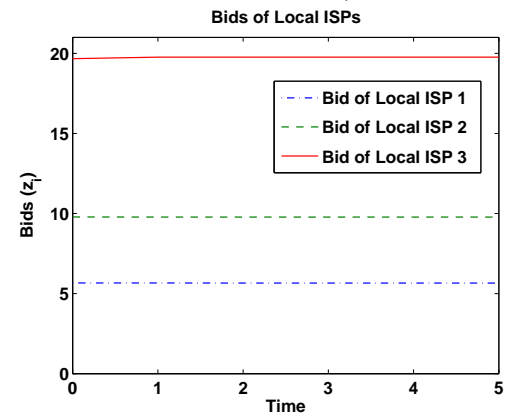

(d) ESA and $\gamma=1$

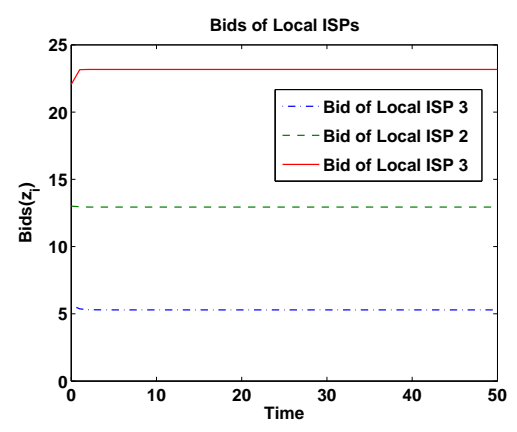

(b) PSA and $\gamma=5$ Bids of Local ISPs

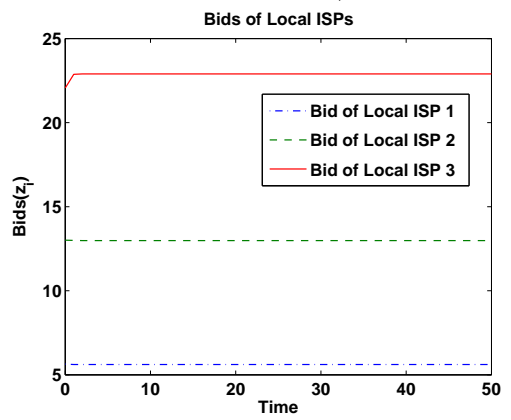

(e) ESA and $\gamma=5$

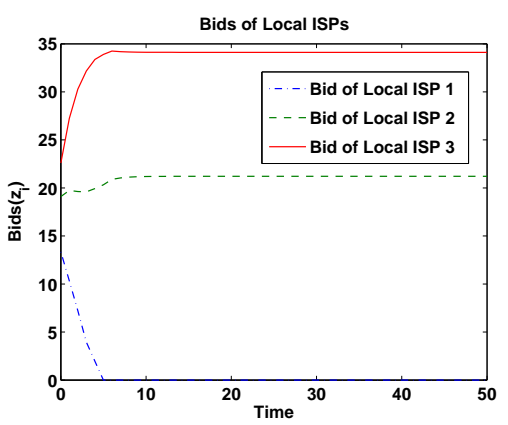

(c) PSA and $\gamma=50$

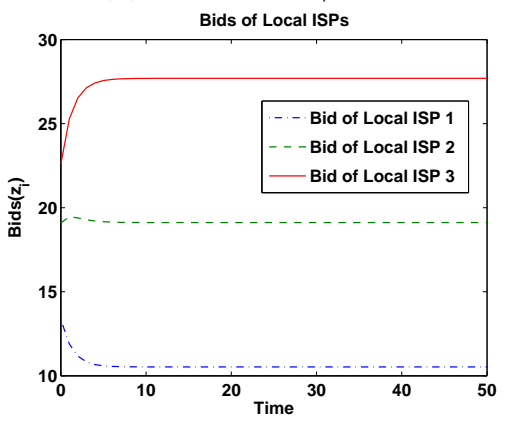

(f) ESA and $\gamma=50$

Fig. 7. Biddings of peers 1,2 and 3 under sufficient bandwidth (a) with Proportional Share Algorithm and $\gamma=1$, (b) with Proportional Share Algorithm and $\gamma=5$, (c) with Proportional Share Algorithm and $\gamma=50$, (d) with Equal Share Algorithm and $\gamma=1$, (e) with Equal Share Algorithm and $\gamma=5$, (f) with Equal Share Algorithm and $\gamma=50$.

The first experiment illustrates the case when ISP has sufficient resource. There are three peers in the network. Each peer has two private links to other peers in the system with capacity $c_{i j}=10$ and a unit price $p_{i j}=1$. Peers 1,2 and 3 have different values of happiness weighting coefficients, $w_{1 j}=10$, $w_{2 j}=15$ and $w_{3 j}=20$ for $j=1,2,3$. ISP provides a link with capacity $n \mathcal{C}=100$ and charges a unit price of $\mathcal{P}=1.5$. The ISP updates the distribution and sends signals to peers every one second. Then every peer computes its own optimal transmission rates routing based on the method in section IV. Figure 7 shows the bidding of each peer during the experiment. The ISP uses PSA in Figure 7(a), (b) and (c) and ESA in Figure 7(d), (e) and (f). The vertical axis shows the biddings of each peer and the horizontal axis shows the time. When the value of $\gamma$ is small, peers offer the same biddings no matter ISP uses PSA or ESA and the biddings converge within a few periods. But when $\gamma=50$, PSA results in a monopolization of resource and ESA does not. Let us explain here. When $\gamma$ increases, the peers 
have larger concerns in the QoS (congestion cost) of the link. They would rather to pay more for better transmission service and they choose to send the traffic through the ISP link and so give higher biddings. Note that the utilities of peers are actually decreasing even they give higher biddings. When the value of $\gamma$ is larger enough, $\gamma=50$ here, the demands in the ISP link is larger than the supply, PSA results in the monopolization of the ISP link and peer 1 can only send traffic through peering links.

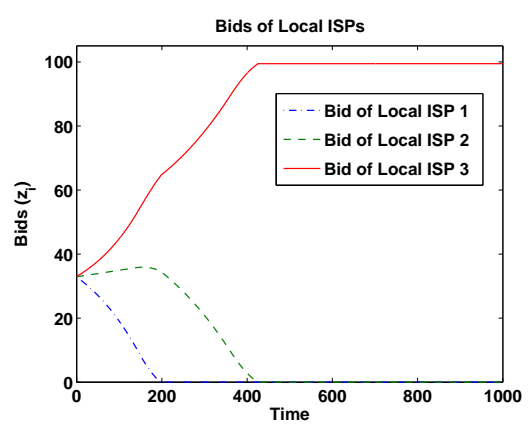

(a) PSA and $\gamma=1$

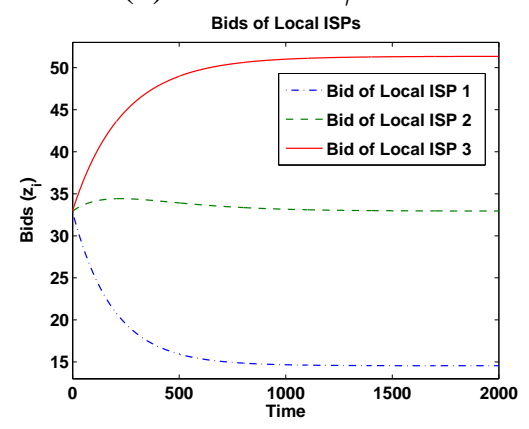

(d) ESA and $\gamma=1$

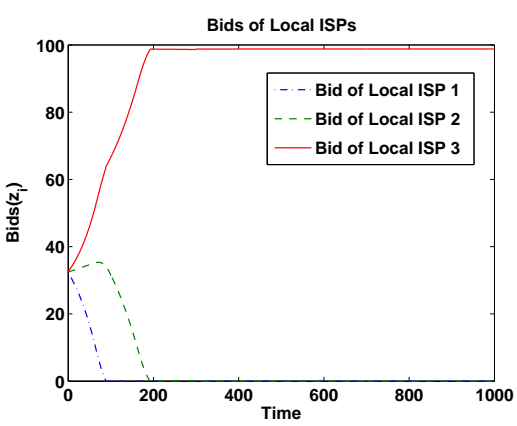

(b) PSA and $\gamma=5$

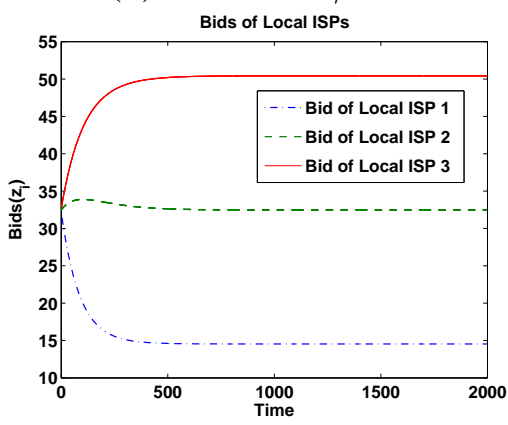

(e) ESA and $\gamma=5$

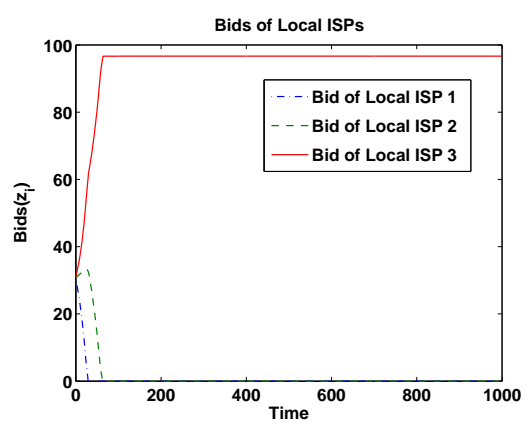

(c) PSA and $\gamma=50$

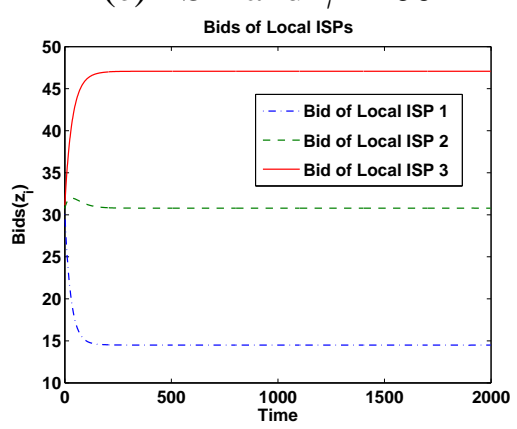

(f) ESA and $\gamma=50$

Fig. 8. Biddings of peers 1,2 and 3 under insufficient bandwidth (a) with Proportional Share Algorithm (b) with Equal Share Algorithm.

The second experiment illustrates the case when ISP has insufficient resource. There are three peers in the network. Each peer has two private links to each other with capacity $c_{i j}=10$ and a unit price $p_{i j}=1$. Peers 1, 2 and 3 have different values of happiness weighting coefficients, $w_{1 j}=100, w_{2 j}=150$ and $w_{3 j}=200$ for $j=1,2,3$. Note that in here the happiness weighting coefficients are much larger than in the previous experiment, meaning that peers do have stronger desires to transmit traffic. Thus keeping the ISP link capacity at the same level leads to an insufficient resource supply. ISP provides a link with capacity $n \mathcal{C}=100$ and charges a unit price of $\mathcal{P}=1.5$. The ISP updates the distribution and sends a signal to peers every one second. Then every peer computes its own optimal transmission rates routing based on the method in section IV. Figure 8 shows the bidding of each peer during the experiment. The ISP uses PSA in Figure 8(a), (b) and (c) and ESA in Figure 8(d), (e) and (f). The vertical axis shows the biddings of each peer and the horizontal axis is the time axis. The experiment runs for 1000 seconds 
for results in Figure 8(a), (b) and (c) and 2000 seconds for results in Figure 8(d), (e) and (f). When ISP uses the PSA policy, no matter when $\gamma=1,5$ or 50 , peer 3 monopolizes the whole ISP link resource and peers 1 and 2 can only send through private links. This is because peer 3 has the largest happiness weighting coefficient, it has the largest tolerance in congestion and bids the largest to the ISP. Under PSA, the ISP then shifts some peer 1's resource to peer 3. The congestion cost in ISP link of peer 1 increases and so peer 1 gives a smaller biddings and is further allocated less resource. This propagates until peer 1's congestion cost in transmitting in ISP link is larger than its happiness and it gives zero bidding. The same happens to peer 2 and finally causes the monopolization of the ISP link resource. So peers 1 and 2 have to shift their traffic to the private links and thus peer 3 finally monopolizes the ISP bandwidth. On the other hand, when ISP uses ESA, the three peers share the ISP link and there is no monopolization.

Remarks: In this section, we show two algorithms for an ISP to distribute its capacity resource among the peers. Both PSA and ESA are efficient, but PSA may result in a monopolized utilization of the resource by a small number of peers. As a contrast, ESA can prevent this undesirable outcome. Note that the monopoly of the ISP resource in PSA can be prevented if some proper upper limits on the peer's bandwidths are set. But since Section IV considers the maximum traffic loading of local ISP and so we do not enforce any upper limits here.

For all simulations, we see that the allocations from ISP and biddings from peers converge. However, the convergence point of the Nash equilibrium is not easy to prove theoretically. We formulate the allocations from ISP and biddings from peers at period $t$ as a function and the output is the new allocations and biddings, i.e. $\left(\mathcal{C}_{1}^{(t+1)}, \ldots, \mathcal{C}_{n}^{(t+1)}, z_{i}^{(t+1)}, \ldots, z_{n}^{(t+1)}\right)=f\left(\mathcal{C}_{1}^{(t)}, \ldots, \mathcal{C}_{n}^{(t)}, z_{i}^{(t)}, \ldots, z_{n}^{(t)}\right)$. The domain and range of this function is in the $2 n$-dimensions space of $[0, n \mathcal{C}]$. But the function is discontinuous at $\overrightarrow{0}$ and so fixed point theorem may not be applicable here.

\section{ISP: Maximization of its Revenue}

In this section, we investigate various approaches for an ISP to maximize its revenue. The revenue of an ISP is the aggregate payments received from $n$ peers for consuming the ISP's link bandwidth. Formally, the ISP's revenue can be expressed as

$$
\mathcal{R}(\mathcal{P})=\sum_{i} \mathcal{P} \cdot z_{i}(\mathcal{P})
$$

In here, $z_{i}(\mathcal{P})$ indicates that one peer's bandwidth consumption on the ISP link is a function of the price $\mathcal{P}$ set by the ISP. It is obvious that, if the price is set too high, peers may switch their traffic to the private links where the service is cheaper. Thus the ISP's revenue reduces. On the other hand, a lower price may attract peers to send more traffic via the ISP, however, too low a price may not ensure an increase in the 
total revenue. These characteristics leave the door open for ISP to search for an optimal price to ensure the maximization of its revenue. Normally, it only makes sense for the ISP to obtain the optimal price in a "blind search" manner. Namely, the ISP randomly proposes a price to see the aggregate bandwidth consumption $\bar{z}$ at the equilibrium point. After finding its revenue at this pricing level, the ISP may adjust its price a little bit to see how it affects its total revenue. With the feedback information the ISP can readjusts its price. However, this type of "local search" method may not ensure the global optimality and it can be very time-consuming. So a natural question arises: is there any effective approach for an ISP to find its optimal price, assuming the ISP can estimate some necessary information about the system, e.g., the happiness weighting coefficients of peers $\left(w_{i j}, \forall i, j\right)$, capacities and unit prices of the private links?

Instead of doing a blind search on the unit price $\mathcal{P}$ to maximize the revenue, we propose an efficient method to estimate the optimal price. This method requires a quick estimation of the aggregate traffic $\bar{z}$ given a fixed ISP link price $\mathcal{P}$, rather than implementing the price and waiting for an equilibrium point to reach. With the estimation of $\bar{z}$, we can easily calculate ISP's revenue for a given $\mathcal{P}$, which provides a possibility for us carry out the pricing search to be introduced shortly.

Before we proceed to the presentation of the pricing search method, let us first illustrate how can an ISP estimate its aggregate bandwidth consumption with a price $\mathcal{P}$. This estimation has the following three assumptions. First, the ISP applies the Equal Share Algorithm (ESA) in resource distribution. As is shown previously, the ESA can avoid the resource monopoly by a single peer. Second, the ISP takes an indiscriminate pricing approach and charges the same unit price of $\mathcal{P}$ to all peers. Third, when the ISP maximizes its revenue, it only considers the case of $z_{i j}>0$ for all $i, j$, which means all peers want to transfer data via the ISP's links.

\section{A. Estimation of aggregate traffic $\bar{z}$ on ISP link}

The estimate of the aggregate bandwidth consumption or the aggregate biddings is complicated. To ease the computation, we will later introduce a variable $k$, which stands for the marginal increases in congestion cost plus unit price. The purpose of the variable $k$ is that one can relate the aggregate bidding $\bar{z}$ in terms of $k$. To find an estimate of the revenue $\mathcal{R}(\mathcal{P})$ with a unit price $\mathcal{P}$, ISP first estimates the value of $k$. Then it can have the estimates of the peers' aggregate biddings and its revenue $\mathcal{R}(\mathcal{P})$. Now, we introduce the procedure for estimating the aggregate biddings of peers $\bar{z}$ with a unit price $\mathcal{P}$.

At the equilibrium point of the peers' biddings under ESA, we have

$$
C_{i}-z_{i}=\frac{n \mathcal{C}-\bar{z}}{n} \quad \text { for } i \in\{1, \ldots, n\}
$$


Substituting Equation (11) into the necessary conditions in Equation (9), we obtain

$$
\begin{aligned}
\frac{w_{i j}}{1+y_{i j}+z_{i j}} & \leq \frac{\gamma}{\left(c_{i j}-y_{i j}\right)^{2}}+p_{i j}, \\
\frac{w_{i j}}{1+y_{i j}+z_{i j}} & =\frac{n^{2} \gamma}{(n \mathcal{C}-\bar{z})^{2}}+\mathcal{P}, \\
\frac{w_{i i}}{1+z_{i i}} & =\frac{n^{2} \gamma}{(n \mathcal{C}-\bar{z})^{2}}+\mathcal{P} .
\end{aligned}
$$

Let us now introduce the variable $k$, which is

$$
\begin{aligned}
k & =\frac{n^{2} \gamma}{(n \mathcal{C}-\bar{z})^{2}}+\mathcal{P}, \\
\text { so } \bar{z} & =n \mathcal{C}-\frac{n \sqrt{\gamma}}{\sqrt{k-\mathcal{P}}}
\end{aligned}
$$

We have two cases to consider: $y_{i j}>0$ and $y_{i j}=0$. First, let us consider the case when $y_{i j}>0$ for all $i \neq j$, Equations (13), (12), and (14) become

$$
\begin{aligned}
& z_{i j}=\frac{w_{i j}}{k}-1-y_{i j} \\
& y_{i j}=c_{i j}-\frac{\sqrt{\gamma}}{\sqrt{k-p_{i j}}} \\
& z_{i i}=\frac{w_{i i}}{k}-1
\end{aligned}
$$

Note that the necessary condition for $y_{i j}>0$ is $k>p_{i j}$. Substituting Equations (17), (18) and (19) into $\bar{z}=\sum_{i} \sum_{j} z_{i j}$, we have:

$$
\begin{aligned}
\bar{z} & =\sum_{i} \sum_{j \neq i}\left(\frac{w_{i j}}{k}-1-\left(c_{i j}-\frac{\sqrt{\gamma}}{\sqrt{k-p_{i j}}}\right)\right)+\sum_{i}\left(\frac{w_{i i}}{k}-1\right)=\sum_{i} \sum_{j} \frac{w_{i j}}{k}-\sum_{i} \sum_{j \neq i} c_{i j}-n^{2}+\sum_{i} \sum_{j \neq i} \frac{\sqrt{\gamma}}{\sqrt{k-p_{i j}}} \\
\bar{z} & =\frac{\bar{W}}{k}-\sum_{i} \sum_{j \neq i} c_{i j}-n^{2}+\sum_{i} \sum_{j \neq i} \frac{\sqrt{\gamma}}{\sqrt{k-p_{i j}}}
\end{aligned}
$$

where $\bar{W}$ is the sum of all happiness weighting coefficients of peers $\left(\bar{W}=\sum_{i} \sum_{j} w_{i j}\right)$.

Now, we can equate (16) and (20) and we have:

$$
n \mathcal{C}-\frac{n \sqrt{\gamma}}{\sqrt{k-\mathcal{P}}}=\frac{\bar{W}}{k}-\sum_{i} \sum_{j \neq i} c_{i j}-n^{2}+\sum_{i} \sum_{j \neq i} \frac{\sqrt{\gamma}}{\sqrt{k-p_{i j}}}
$$




$$
\begin{aligned}
n \mathcal{C}+\sum_{i} \sum_{j \neq i} c_{i j}+n^{2} & =\frac{\bar{W}}{k}+\frac{n \sqrt{\gamma}}{\sqrt{k-\mathcal{P}}}+\sum_{i} \sum_{j \neq i} \frac{\sqrt{\gamma}}{\sqrt{k-p_{i j}}} \\
\overline{\mathcal{C}}_{0}+n^{2} & \approx \frac{\bar{W}}{k}+\frac{n \sqrt{\gamma}}{\sqrt{k-\mathcal{P}}}+\frac{n(n-1) \sqrt{\gamma}}{\sqrt{k-p_{a v}}}
\end{aligned}
$$

where $\overline{\mathcal{C}}_{0}=n \mathcal{C}+\sum_{i} \sum_{j \neq i} c_{i j}$ is the aggregate capacities in the system, $p_{a v}=\frac{1}{n(n-1)} \sum_{i} \sum_{j \neq i} p_{i j}$ is the mean of $p_{i j}$. We have the estimate of Equation (21) if the variance of unit prices of private links is small.

Having some information of the happiness weighting coefficients and capacities and unit prices of private links, the ISP can estimate the value of $k$ using Equation (21), and then estimate the aggregate biddings $\bar{z}$ with Equation (16) and its revenue $\mathcal{R}(\mathcal{P})$ with Equation (10).

Now, let us consider the second case when $y_{i j}=0$ for all $i \neq j$, Equations (12) and (13) give a necessary condition of $k$, which is

$$
k=\frac{w_{i j}}{1+z_{i j}} \leq \frac{\gamma}{c_{i j}^{2}}+p_{i j}
$$

Substituting Equations (11), (13), (14) and (16) into $\bar{z}=\sum_{i} \sum_{j} z_{i j}$, we have:

$$
\begin{aligned}
n \mathcal{C}-\frac{n \sqrt{\gamma}}{\sqrt{k-\mathcal{P}}} & =\sum_{i} \sum_{j}\left(\frac{w_{i j}}{k}-1\right)=\frac{\bar{W}}{k}-n^{2} \\
n \mathcal{C}+n^{2} & =\frac{\bar{W}}{k}+\frac{n \sqrt{\gamma}}{\sqrt{k-\mathcal{P}}}
\end{aligned}
$$

Again, ISP can compute the value of $k$ with Equation (23) with cubic formula [4], then estimates the aggregate biddings $\bar{z}$ with Equation (16) and its revenue $\mathcal{R}(\mathcal{P})$ with Equation (10).

Remarks: We presented the procedure for an ISP to estimate the aggregate demand of traffic from its customers and altogether its revenue, with a fixed unit price of its bandwidth $\mathcal{P}$.

\section{B. Optimal Pricing Search Method}

Before going into the pricing search method, we first provide an intuition of the method. When the unit price of the ISP link $(\mathcal{P})$ is small, an increase in the price only reduces the aggregate biddings of the peers $\bar{z}$ slightly. So the ISP has an increase of revenue as the loss in the decreasing bidding is covered by the gain in the increment of unit price. The increase of revenue vanishes when the marginal point is reached, where the gain from increasing unit price can no longer cover the loss from the decreasing demand.

The proposed pricing search method is divided into two phases. The purpose of phase one is to find a feasible range of the optimum unit price with the help of the estimation of aggregate traffic in section 
VI-A. Phase two aims at reducing the size of the feasible range obtained in phase one by trisection method. The method is depicted as follows:

\section{Pricing Search Method:}

1. ISP initiates a step size $\sigma$ and a threshold $\delta$.

2. /* Phase 1: */

3. while (1) \{

4. $\quad$ ISP computes four unit prices $\mathcal{P}_{1}=\sigma, \mathcal{P}_{2}=2 \mathcal{P}_{1}, \mathcal{P}_{3}=2 \mathcal{P}_{2}$ and $\mathcal{P}_{4}=2 \mathcal{P}_{3}$.

5. ISP computes four revenues $\mathcal{R}\left(\mathcal{P}_{1}\right), \mathcal{R}\left(\mathcal{P}_{2}\right), \mathcal{R}\left(\mathcal{P}_{3}\right)$ and $\mathcal{R}\left(\mathcal{P}_{4}\right)$.

6. $\quad$ if $\left(\mathcal{R}\left(\mathcal{P}_{3}\right)>\mathcal{R}\left(\mathcal{P}_{4}\right)\right)$

7. break; / go to phase $2 \star /$

8. else

9. $\sigma=2 \sigma / \star$ go back to phase 1 */

10. \} / * termination of while-loop of phase $1 * /$

11. /* Phase 2: */

12. while (1) \{

13. if $\left(\mathcal{P}_{4}-\mathcal{P}_{1}<\delta\right)$

14. $\quad$ return $\mathcal{P}_{1}$; break;

15. else if $\left(\mathcal{R}\left(\mathcal{P}_{2}\right)<\mathcal{R}\left(\mathcal{P}_{3}\right)\right)$

16. update $\mathcal{P}_{1}=\mathcal{P}_{2}, \mathcal{P}_{2}=\mathcal{P}_{3}$ and $\mathcal{P}_{3}=\frac{\mathcal{P}_{2}+\mathcal{P}_{4}}{2}$.

17. $\quad$ else if $\left(\mathcal{R}\left(\mathcal{P}_{2}\right)>\mathcal{R}\left(\mathcal{P}_{3}\right)\right)$

18. $\quad$ update $\mathcal{P}_{4}=\mathcal{P}_{3}, \mathcal{P}_{3}=\mathcal{P}_{2}$ and $\mathcal{P}_{2}=\frac{\mathcal{P}_{1}+\mathcal{P}_{3}}{2}$.

19. $/$ / termination of while-loop of phase 2 */

Initially, ISP chooses a step size $\sigma$ and a threshold $\delta$. In phase one, the ISP finds four unit prices based on $\sigma$ such that $\mathcal{P}_{1}=\sigma, \mathcal{P}_{2}=2 \mathcal{P}_{1}, \mathcal{P}_{3}=2 \mathcal{P}_{2}$ and $\mathcal{P}_{4}=2 \mathcal{P}_{3}$. Then it estimates the revenues at these four prices, $\mathcal{R}\left(\mathcal{P}_{1}\right), \mathcal{R}\left(\mathcal{P}_{2}\right), \mathcal{R}\left(\mathcal{P}_{3}\right)$ and $\mathcal{R}\left(\mathcal{P}_{4}\right)$. If $\mathcal{R}\left(\mathcal{P}_{3}\right)>\mathcal{R}\left(\mathcal{P}_{4}\right)$, it means that we have found the feasible range $\left[\mathcal{P}_{1}, \mathcal{P}_{4}\right]$ and go to phase two. Otherwise, if $\mathcal{R}\left(\mathcal{P}_{3}\right) \leq \mathcal{R}\left(\mathcal{P}_{4}\right)$, we update $\sigma=2 \sigma$ and go back to phase one again. The stopping criteria bases on the assumption that a local optimal price is also the global optimal price. So a decrease in the revenue when price is increasing notes the ending of phase one.

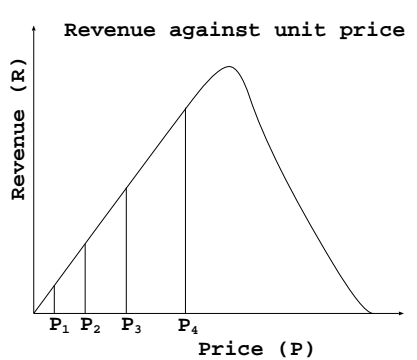

(a)

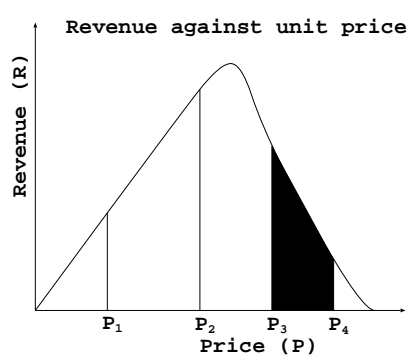

(b)

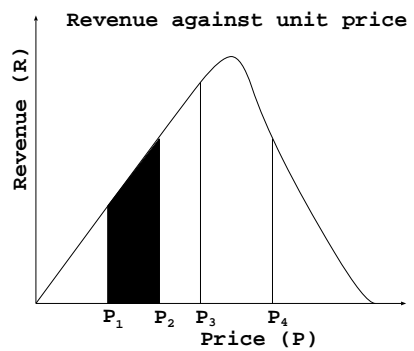

(c)

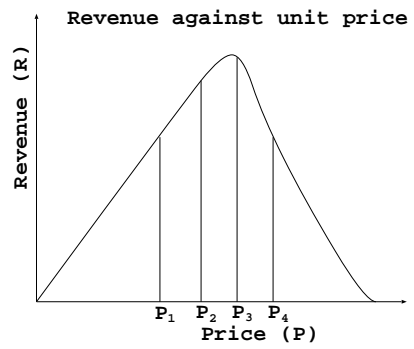

(d)

Fig. 9. (a) ISP finds four unit prices and estimates the revenues at the four prices. (b) $\mathcal{R}\left(\mathcal{P}_{3}\right)>\mathcal{R}\left(\mathcal{P}_{4}\right)$, stands the end of phase one and we go to phase two. Moreover, since $\mathcal{R}\left(\mathcal{P}_{2}\right)>\mathcal{R}\left(\mathcal{P}_{3}\right)$, we prune out the region $\left[\mathcal{P}_{3}, \mathcal{P}_{4}\right]$. (c) Update $\mathcal{P}_{4}=\mathcal{P}_{3}, \mathcal{P}_{3}=\mathcal{P}_{2}$ and $\mathcal{P}_{2}=\frac{\mathcal{P}_{1}+\mathcal{P}_{3}}{2}$. Since $\mathcal{R}\left(\mathcal{P}_{2}\right)<\mathcal{R}\left(\mathcal{P}_{3}\right)$, we prune out the region $\left[\mathcal{P}_{1}, \mathcal{P}_{2}\right]$. (d) Update $\mathcal{P}_{1}=\mathcal{P}_{2}$, $\mathcal{P}_{2}=\mathcal{P}_{3}$ and $\mathcal{P}_{3}=\frac{\mathcal{P}_{2}+\mathcal{P}_{4}}{2}$. 
In phase two, we aim at reducing the feasible range obtained in last phase to be within our threshold by trisection method. We compare the two revenues $\mathcal{R}\left(\mathcal{P}_{2}\right)$ and $\mathcal{R}\left(\mathcal{P}_{3}\right)$. If $\mathcal{R}\left(\mathcal{P}_{2}\right) \leq \mathcal{R}\left(\mathcal{P}_{3}\right)$, the optimum unit price is in between $\left[\mathcal{P}_{2}, \mathcal{P}_{4}\right]$. This is again based on the assumption that a local optimal price is also the global optimal price. So we update $\mathcal{P}_{1}=\mathcal{P}_{2}$ and $\mathcal{P}_{2}=\mathcal{P}_{3}$ and $\mathcal{P}_{3}=\frac{\mathcal{P}_{2}+\mathcal{P}_{4}}{2}$. If $\mathcal{R}\left(\mathcal{P}_{2}\right)>\mathcal{R}\left(\mathcal{P}_{3}\right)$, the optimum unit price is in the range $\left[\mathcal{P}_{1}, \mathcal{P}_{3}\right]$, and we update $\mathcal{P}_{4}=\mathcal{P}_{3}, \mathcal{P}_{3}=\mathcal{P}_{2}$ and $\mathcal{P}_{2}=\frac{\mathcal{P}_{1}+\mathcal{P}_{3}}{2}$. Phase two ends when the size of the range, $\mathcal{P}_{4}-\mathcal{P}_{1}<\delta$ (the threshold). Figure 9 illustrates the procedure of the search. In figure 9(a), ISP estimates the revenues at four prices $\mathcal{P}_{1}, \mathcal{P}_{2}, \mathcal{P}_{3}$ and $\mathcal{P}_{4}$. In figure 9(b), $\mathcal{R}\left(\mathcal{P}_{3}\right)>\mathcal{R}\left(\mathcal{P}_{4}\right)$ marks the end of phase one and enters phase two. As $\mathcal{R}\left(\mathcal{P}_{2}\right)>\mathcal{R}\left(\mathcal{P}_{3}\right)$, we prune out the unfeasible region $\left[\mathcal{P}_{3}, \mathcal{P}_{4}\right]$. In figure $9(\mathrm{c})$, we update the prices and find that $\mathcal{R}\left(\mathcal{P}_{2}\right)<\mathcal{R}\left(\mathcal{P}_{3}\right)$, so we prune out the unfeasible region $\left[\mathcal{P}_{1}, \mathcal{P}_{2}\right]$. Figure $9(\mathrm{~d})$ shows the remaining feasible region and updated price of figure $9(\mathrm{c})$.

Remarks: We introduced a method for an ISP to quickly search for the optimal price in which its revenue is maximized. This optimal price $\mathcal{P}^{*}$ is only computed once and the ISP will use this price $\mathcal{P}^{*}$ in all the iterations of resource allocation.

\section{Illustration of Optimal Pricing Policy}

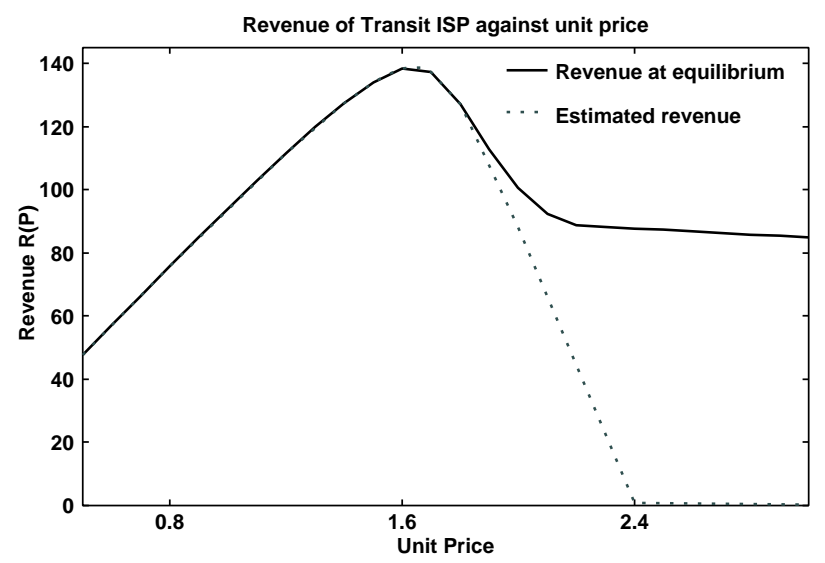

Fig. 10. ISP's revenue at equilibrium and its estimated revenue with different unit price.

To illustrate the above procedure, let us consider an experiment and show how the ISP can find the optimal unit price to maximum its revenue. We have a network with five peers. Each peer has four peering links connecting to the other peers in the system with capacity $c_{i j}=10$ and a unit price $p_{i j}=$ 1. Peers have different values of happiness weighting coefficients, $w_{1 j}=18, w_{2 j}=19, w_{3 j}=20$, $w_{4 j}=21, w_{5 j}=22$ for $j \in\{1, \ldots, 5\}$. The mapping variable $\gamma=1$. ISP provides a link with capacity $n \mathcal{C}=100$ and it applies the Equal Share Algorithm to compute the resource allocation. It then sends a signal to peers and updates the distribution every one second. Figure 10 shows the revenue of the ISP with different unit prices of the ISP link. The vertical axis shows the actual revenue and 
estimated revenue of the ISP and the horizontal axis shows the unit price of the link. The actual revenue is computed at the equilibrium point where the biddings of from the peers converge. The estimated revenue is computed using the algorithm proposed in Section VI-B and Equation (21). The experiment shows that both the proposed algorithm and equation can correctly estimate the optimal unit price at $\mathcal{P}^{*}=1.6$. In conclusion, the ISP can do an offline pricing search to find the optimal value of $\mathcal{P}^{*}$ for the maximization of revenue.

\section{Issue of Network Scaling}

In this section, we seek to further explore the regime when we scale up the network, i.e., increasing the number of peers or $n \rightarrow \infty$. By studying the behaviors of ISP and peers in this many user regime, it allows us to obtain a fundamental understanding of the relationship between ISPs at different tiers. Firstly, we analyze how the peers' biddings can affect the decision of ISP, i.e., the optimal price to maximize its total revenue. Furthermore, the study also enables us to ask (and answer) some interesting yet important questions, such as whether the peers have incentive to set up peering relationship between themselves, and whether the service provider benefits from upgrading the network (i.e., upgrade the backbone capacity) to admit new users so as to increase its revenue.

Our analysis is based on the results previously obtained in Section VI. Equations (21)(23) specify an estimate of the aggregate demand on the ISP transit services. If the ISP knows the following information, e.g., an estimate of the happiness coefficients of all peers, pricing policies and capacities of the peering links, then it has an opportunity to infer its optimal pricing strategy to maximize its revenue. Although the pricing policy and peers' capacities are generally regarded as business confidential information which is difficult to obtain, a rough prediction of the distribution on these information can help the ISP to make the marketing decision. Another application of Equations (21)(23) is to predict how the number of peers affects the ISP's maximal revenue as well as its marginal profit. The answer to this question gives us an important insight into the evolution of future's Internet. For the completeness of presentation, the situation we consider can be categorized into two cases: a) there are no peering links between peers. b) peers do set up peering relationships which result in a meshed peering network.

\section{A. Network Scaling without peering links between peers}

When there is no peering link between peers, peers can only rely on the ISP link to communicate with each other. An interesting question is whether the ISP can arbitrarily monopolize the market? Or equivalently, how high a price can the ISP charge to maximize its profit?

From the analysis in Section VI, we know that when there is no peering link between peers, i.e., 
$y_{i j}=0$, Equation(23) holds, which can be approximated as:

$$
n \mathcal{C}+n^{2}=\frac{n^{2} \bar{w}}{k}+\frac{n \sqrt{\gamma}}{\sqrt{k-\mathcal{P}}}
$$

wherein $\bar{w}$ can be estimated as the average happiness coefficient of all peers. The necessary condition for this equation to hold, i.e., there is no traffic transfer on peering links, is $k \leq \frac{\gamma}{c_{i j}^{2}}+p_{i j}$. This condition satisfies when there is no peering links $\left(c_{i j}=0\right)$, or the expense for transfer on peering links is too high. Applying similar approaches we have discussed in Section VI, one can calculate the ISP's revenue under different values of $\mathcal{P}$ and $n$. Figure 11 illustrates ISP's revenue as a function of $\mathcal{P}$, for different numbers of peers. Note that in this attempt we assume the ISP does not upgrade the link capacity even when there are more peers joining the network. So we keep $n \mathcal{C}=100$ for all $n$.

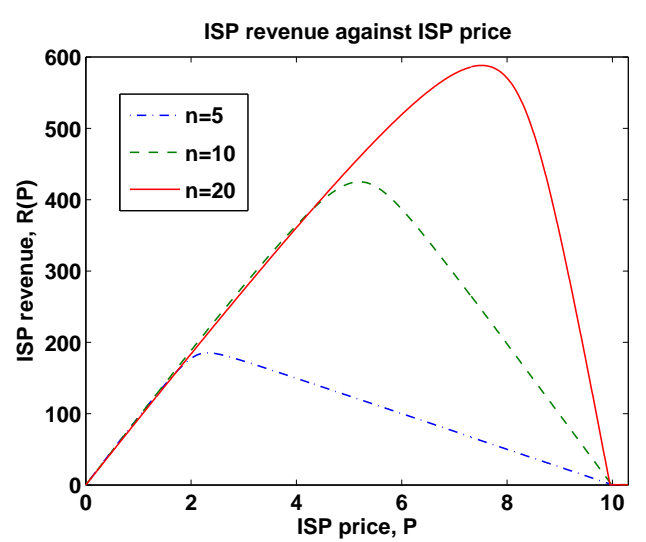

(a)

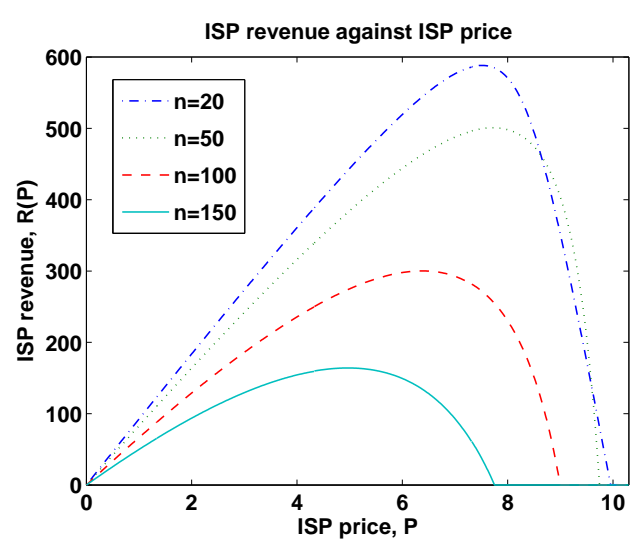

(b)

Fig. 11. Without peering links: ISP's revenue v.s. $\mathcal{P}$ under different network scales. ISP link capacity is kept constant: $n \mathcal{C}=100(\bar{w}=10)$. ISP's maximal revenue (a) increases, (b) decreases as the number of peers $n$ increases.

Some observation is made here. Firstly, given a particular value of $n$, we can always find out a unique optimal price $\mathcal{P}^{*}$ that maximizes the ISP's revenue, which is a verification of the results presented in Section VI. The ISP can always increase its profit by raising the service charge from a zero (free) level. Note that as the charge $\mathcal{P}$ exceeds a threshold, the ISP's revenue starts to decrease and eventually falls to zero. In this case, no peer would choose to transmit through the ISP link due to the high price. Another observation made here is the impact of network scale on the ISP's profit. When the scale of the network is small, i.e. $n \leq 20$, the ISP is able to receive more revenue as more peers require connecting service, which is due to the increase in the service demand. However, when the number of peers exceeds a threshold ( $n=20$ is a threshold in this illustration), the ISP's maximal revenue tends to decrease. This counter-intuitive fact can be explained by the constraint on the limited bandwidth. Since more peers are 
competing for a fixed ISP capacity, the bandwidth allocation for each peer tends to be very limited. The poor quality of service thus prevents them from utilizing the ISP link. Worse yet, when $n$ is sufficiently large, the ISP will not receive any revenue at all. This result verifies again that the ISP's policy should adapt to the changes of the market demand.

In contrast to the case when the capacity of ISP link is kept fixed, we illustrate via Figure 12 when the capacity of the ISP link increases proportionally to the number of peers in the network. Thus ISP has a total capacity of $n \mathcal{C}$ where $C=100$ is a constant. Figure 12(a) illustrates ISP's revenue as a function of its announced price, under different network scales. This figure shows that if the ISP upgrades its link capacity proportionally to the number of peers, it can always achieve an increasing revenue as $n$ increases, which is a contrast to the fixed-capacity case. Further, the revenue is able to increase faster than $n$ does. Figure 12(b) plots the relationship between ISP's maximal revenue per bandwidth $\left(\bar{z} \mathcal{P}^{*} / n \mathcal{C}\right)$ and the network size $n$. As showed, the ISP's marginal profit keeps increasing as $n$ grows, which implies that the ISP can benefit from upgrading its network, a good news for the service providers.

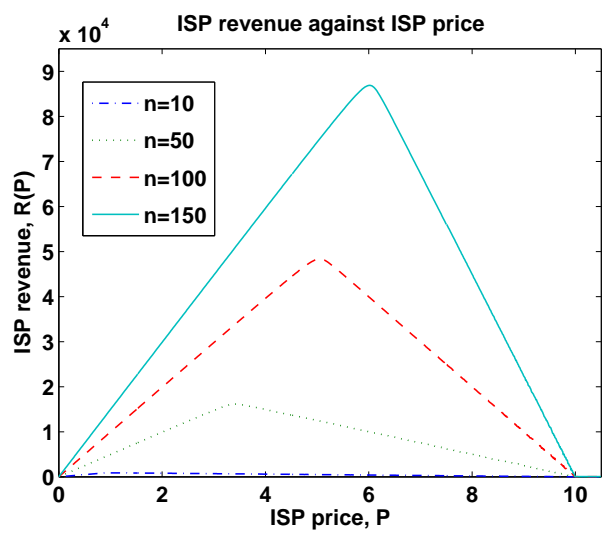

(a)

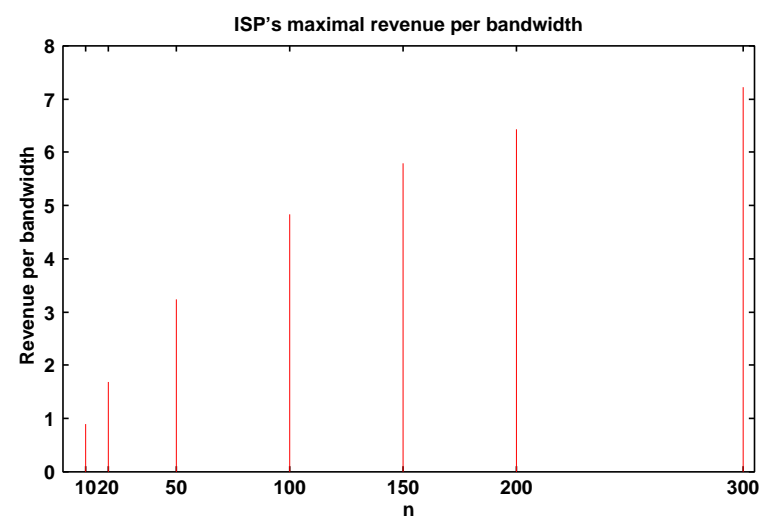

(b)

Fig. 12. Without peering links, ISP's revenue as the ISP link capacity grows proportionally: (a) ISP revenue v.s. $\mathcal{P}$ under different network sizes. (b) ISP's maximal revenue per bandwidth grows as more peers join the market.

\section{$B$. Network scaling with peering links between peers}

In the previous section, we have analyzed the relationship between ISP's revenue and peers' population when there is no outside competition from the peers. In here, we further explore the scenarios when there are peering links between peers, especially, traffic transmission does carry out on the peering links. The situation occurs when peers upgrade the capacities of peering links, or offer the service at a lower price, thus attracting more traffic to go through the peering links. We seek to answer the following question: does the conclusion draw in Section VII-A still hold under this competitive framework? 
In Section VI, we have showed that when $y_{i j}>0$, Eq. (21) holds, subjected to the condition that $k \geq p_{i j}+1 / c_{i j}^{2}$ and $\mathcal{P}+n^{2} / C^{2}<k<w_{i j}$. By solving this equation, one can calculate the ISP's revenue for different values of $\mathcal{P}$ and $n$. Similarly, we first explore the case when ISP does not increase its link capacity even if the number of peers increases, i.e., $n \mathcal{C}=100$ remains a constant. And we perform the analysis under the settings $w_{i j}=10, p_{i j}=1, c_{i j}=10$ for all $i, j$. Figure 13 illustrates how the number of peers $n$ affects ISP's maximal revenue.

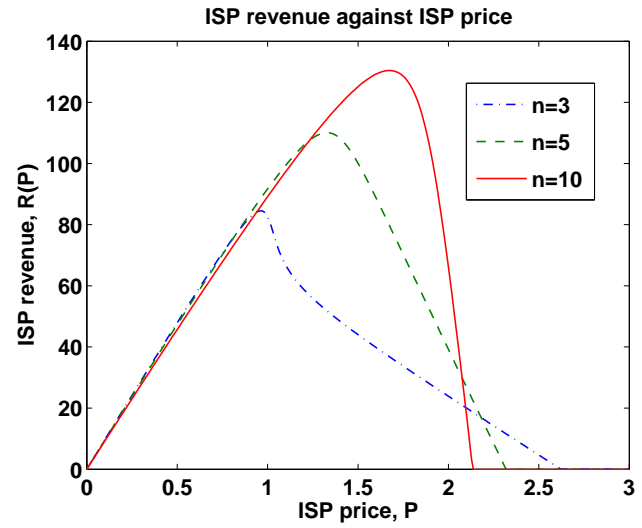

(a)

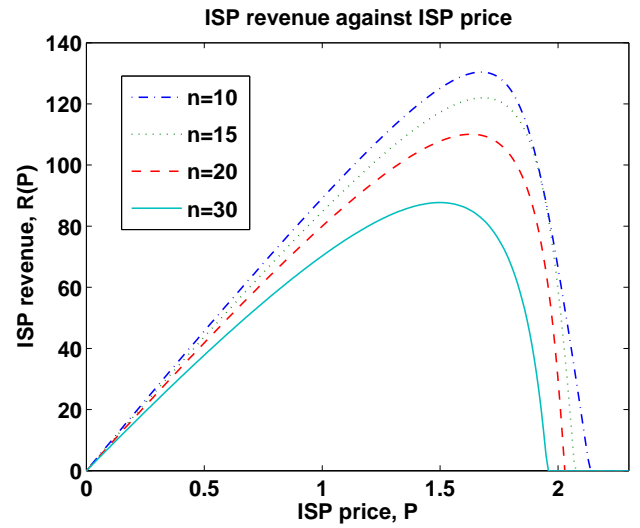

(b)

Fig. 13. With peering links: ISP's revenue v.s. $\mathcal{P}$ under different network scales. ISP link capacity is kept constant: $n \mathcal{C}=100$ $(\bar{w}=10)$. ISP's maximal revenue (a) increases (b) decreases as the number of peers $n$ increases.

Some observation is made here. When the competition from the peering links is introduced, the ISP's maximal revenue increases initially, but decreases rapidly as the number of peers grows, where $n=5$ is the threshold. This is because as $n$ increases, the available ISP bandwidth allocated to each peer is decreasing when the ESA is performed. Peers have to bear a large congestion cost due to the limited bandwidth, which forces them to go through the peering links. This fact provides an indication that ISPs do have incentive to upgrade their network to satisfy the increasing demand for the ISP bandwidth.

Similarly, we also analyze the ISP's benefit from increasing the link capacity. We assume that the ISP's link capacity grows proportionally to the number of peers in the network, i.e., the ISP has a total capacity of $n \mathcal{C}$, where $\mathcal{C}=100$. Figure 14 illustrates the relationship between ISP's maximal revenue and the network size. The first observation is that as the number of peers grows, the ISP's maximal revenue keeps increasing. From Figure 14(b), we can also see that the revenue increasing rate is faster than that of $n$. This confirms that ISP has the incentive to improve the network infrastructures so as to increase its revenue. However, when compared to the case without peering links, the revenue increasing rate becomes lower as the network size increases. Especially, when $n>200$, the increase in profit per bandwidth is very small. This is due to the fact that peers set up peering links to form a mesh-network. It 


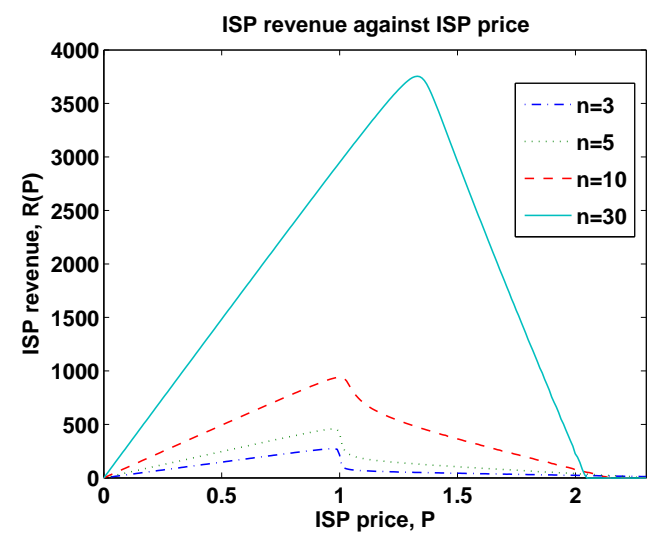

(a)

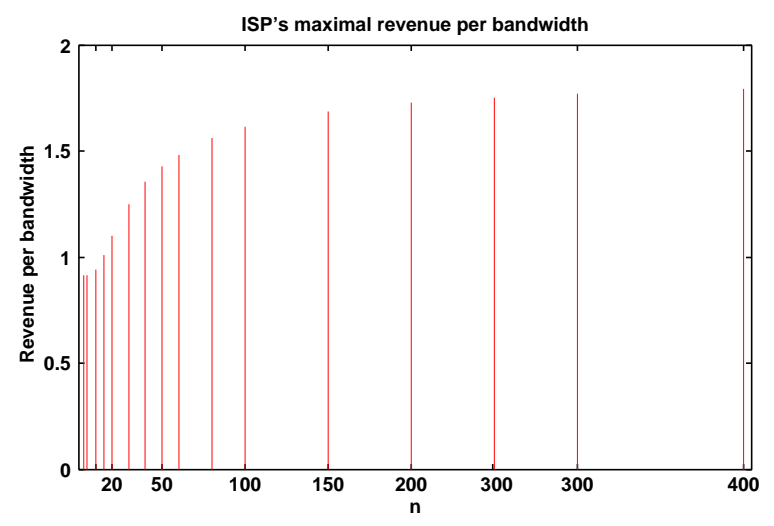

(b)

Fig. 14. With peering links, ISP's revenue when ISP link capacity grows proportionally: (a) ISP revenue versus $\mathcal{P}$ under different network sizes. (b) ISP's maximal revenue grows as more peers join.

is the competition that brings down the ISP's marginal profit. It is worthwhile to mention that in practice, it may be difficult for all peers to form a fully meshed network among themselves. This may be due to the geographical constraint that some peers are located very far apart, or may be due to the legal regulations. Thus there are still great opportunities for ISPs to gain by upgrading the network infrastructures.

\section{Related Work}

Let us present a brief review of some related work. There are a large model of works about Internet pricing [13], [18], [10], [11], but they are mostly about customer pricing strategy, or to provide differentiated service. On the other hand, our work focus on the "interaction" between the major ISP and local ISPs. In [2], authors investigated the revenue maximization and scalability of a service provider. Their work showed that there is rationale for the service provider to upgrade its capacity. But their model is different from ours in two ways. There is only one common link in the ISP and each peer considers the congestion cost of that common link. Our model differs from them in a sense that we allow a more realistic representation of today's Internet, that is, we allow peers to have private links so as to reduce their cost. In [17] proposed a model consisting of local and transit ISPs. They showed that optimal strategy of local ISPs is to play "cooperatively" by threat. Our work found the conditions for every peer to achieve its maximum utility.

\section{Conclusion}

In this paper, we investigate the interplay between a higher tier ISP and $n$ local ISPs (which we terms as peers). A peer has a connection to the ISP, and possibly connected to other peers with some private links. Each peer needs to determine the appropriate amount of traffic via the ISP's link and the private 
links so as to maximize its utility and it pays the ISP on a monthly base. The ISP, on the other hand, needs to perform proper resource allocation so as to avoid resource monopoly and to maximize its revenue. We show the necessary and boundary conditions for the traffic rate vectors of a peer to obtain the maximum utility. We present two distributed algorithms for the ISP to do the resource allocation. Both distributed algorithms converge quickly in case the ISP has sufficient resource. We show how the ISP can estimate its revenue with a unit price and we also propose a procedure on how an ISP can obtain the optimum unit price so as to maximize its revenue. Finally, we show that the ISP can obtain a higher revenue by upgrading its capacity when we scale up the network. The proposed methodology provides us a systematic way to determine pricing and resource allocation even when the ISP and peers interact with each other. It is interesting to extend this model to multiple ISPs since one has to consider the interaction and competitions among these ISPs as well. This will be in our future work.

Acknowledgments: We wish to thank the anonymous referees for their helpful and insightful comments.

\section{REFERENCES}

[1] K. M. Abadir and J. R. Magnus. Matrix Algebra. Cambridge University Pres, 2005.

[2] T. Basar and R. Srikant. Revenue-maximizing pricing and capacity expansion in a many-user regime. In IEEE INFOCOM, pages 1556-1563, New York, June 2002.

[3] D. P. Bertsekas and J. N. Tsitsiklis. Parallel and Distributed Computation. Prentice Hall, 1989.

[4] G. Cardano. Cubic formula from the Mathworld.

[5] L. He and J. Walrand. Pricing and revenue sharing strategies for internet service providers. In IEEE INFOCOM, pages 942-951, Miami, March 2005.

[6] L. He and J. Walrand. Pricing differentiated internet services. In IEEE INFOCOM, pages 195-204, Miami, March 2005.

[7] G. Huston. Interconnection, peering and settlements., 1998.

[8] F. Kelly. Charging and rate control for elastic traffic. In European Trans. on Telecommunications, volume 8, pages 33-37, 1997.

[9] J.-J. Laffont, S. Marcus, and P. R. J. Tirole. Internet interconnection and the off-net cost pricing principle. In Institut d'economic Industrielle (IEDI), Mimeo, France, April 2001.

[10] P. Marbach. Pricing differentiated services networks: Bursty traffic. In IEEE INFOCOM, pages 650-658, Alaska, March 2001.

[11] P. Marbach. Priority service and max-min fairness. In IEEE INFOCOM, volume 11, pages 733-746, New York, March 2002.

[12] W. B. Norton. A business case for isp peering. In $N A N O G$, Available at http://www.equinix.com/pdf/whitepapers/Business_case.pdf.

[13] A. M. Odlyzko. The economics of the internet: Utility, utilization, pricing, and quality of service. In AT\&T Research Lab, Tech Report, September 1998.

[14] A. M. Odlyzko. Pricing and architecture of the internet: Historical perspectives from telecommunications and transportation. In The 32nd Research Conference on Communication, Information and Internet Policy, March 2004.

[15] M. J. Osborne and A. Rubinstein. A Course in Game Theory. The MIT Press, 1957.

[16] S. S. Rao. Engineering Optimization: Theory and Practice Third Edition. A Wiley-Interscience Publication, 1996.

[17] S. Shakkottai and R. Srikant. Economics of network pricing with multiple isps. In IEEE INFOCOM, volume 1, pages 184-194, Miami, March 2005.

[18] S. Shenker, D. Clark, D. Estrin, and S. Herzog. Pricing in computer networks: Reshaping the research agenda. In ACM SIGCOMM Computer Communication Review, volume 26, pages 19-43, New York, USA, 1996.

[19] J. W. Stewart. BGP4 : Inter-Domain Routing in the Internet. Addison Wesley, 1999. 
[20] F. Wang and L. Gao. On inferring and characterizing internet routing policies. In Proceedings of the 3rd ACM SIGCOMM conference on Internet measurement, pages 15-26, October 2003. 